\title{
The linear two-dimensional stability of inviscid vortex streets of finite-cored vortices
}

\author{
By D. I. MEIRON $\dagger$, P. G. SAFFMAN AND J. C. SCHATZMAN \\ Applied Mathematics, California Institute of Technology, Pasadena, Cal 91125
}

(Received 28 November 1983 and in revised form 17 May 1984)

The stability of two-dimensional infinitesimal disturbances of the inviscid Kármán vortex street of finite-area vortices is reexamined. Numerical results are obtained for the growth rate and oscillation frequencies of disturbances of arbitrary subharmonic wavenumber and the stability boundaries are calculated. The stabilization of the pairing instability by finite area demonstrated by Saffman \& Schatzman (1982) is confirmed, and also Kida's (1982) result that this is not the most unstable disturbance when the area is finite. But, contrary to Kida's quantitative predictions, it is now found that finite area does not stabilize the street to infinitesimal two-dimensional disturbances of arbitrary wavelength and that it is always unstable except for one isolated value of the aspect ratio which depends upon the size of the vortices. This result does agree, however, with those of a modified version of Kida's analysis.

\section{Introduction}

We consider the stability of two-dimensional infinitesimal disturbances of the steady inviscid incompressible flow produced by infinite rows of finite-cored vortices. The undisturbed flow in a frame of reference moving with the vorticity is described by a stream function $\Psi(x, y)$ with the property that $\Psi(x+l, y) \equiv \Psi(x, y)$, and $\Psi(x, \infty)=U_{1} y, \Psi(x,-\infty)=U_{2} y$. There are two cases: (i) wake-type flows in which $U_{1}=U_{2}=U$, say; and (ii) mixing-layer-type flows with $U_{2}=-U_{1}=U$. In the first case there is no net vorticity; the canonical flow is that of the Kármán vortex street of two infinite straight parallel staggered rows of point vortices of equal and opposite circulation $\Gamma$, with separation $l$ parallel to the rows, distance $h$ between the rows, and each vortex opposite the midpoint of vortices in the other row. In the second case, there is net vorticity and the canonical example is a single straight infinite row of point vortices of equal circulation $\Gamma$ separated by distance $l$.

Infinitesimal disturbances to these flows are described by a perturbation $\epsilon \Psi^{\prime}(x, y, t)$ to the stream function, where $\epsilon$ is infinitesimal and by Floquet or Bloch wave theory the perturbation is a sum of modes of the form

$$
\Psi^{\prime}=\mathrm{e}^{\sigma t} \mathrm{e}^{2 \pi \mathrm{i} p x / l} \sum_{n=-\infty}^{\infty} \phi_{n}(y) \mathrm{e}^{2 \pi \mathrm{i} n x / l} .
$$

It follows from the Euler equations that the perturbation satisfies the linear partial differential equation

$$
\nabla^{2} \Psi_{t}^{\prime}+\frac{\partial\left(\nabla^{2} \Psi^{\prime}, \Psi\right)}{\partial(x, y)}+\frac{\partial\left(\nabla^{2} \Psi, \Psi^{\prime}\right)}{\partial(x, y)}=0
$$

$\dagger$ Present address: Department of Mathematics, University of Arizona, Tueson.

$\ddagger$ Present address: Chevron Oil Field Research, La Habra, California. 
Substitution of (1.1) into (1.2) and requiring that $\Psi^{\prime}$ be bounded gives an eigenvalue problem for the unknown growth rate $\sigma$ as a function of the undisturbed flow and $p$. Notice that $p$ is an arbitrary real number, which clearly without loss of generality may be taken in the range $0 \leqslant p \leqslant 1$ or $-\frac{1}{2} \leqslant p \leqslant \frac{1}{2}$, since adding an integer to $p$ is simply equivalent to relabelling the eigenvector $\phi_{n}$. If $p=0$ the disturbance has the same spatial period $l$ as the undisturbed flow, and will be called a superharmonic disturbance. If $p \neq 0$ the disturbance has in general a component of wavelength $l / p$ or $l /(1-p)$, which is larger than $l$, and will be called subharmonic. The case $p=\frac{1}{2}$, in which the disturbance wavelength is double that of the undisturbed flow, has attracted particular attention in flows of mixing-layer type, where it is called the pairing instability.

If $\sigma$ is pure imaginary for all values of $p$, the disturbance does not grow with time and the flow is said to be stable. (Strictly speaking, it is only stable to infinitesimal disturbances, but the behaviour of finite-amplitude disturbances is an open and difficult question and will not be considered here.) If $\sigma$ has a positive real part for some value of $p$, then the flow is unstable.

The case of point vortices has been investigated in detail by many workers; Lord Kelvin and J. J. Thomson studied the single row in the form of a circle (for a complete treatment see Havelock 1931). Kármán worked out the stability of the double row; Lamb (1932) provides an account of this work and describes the stability of the single row, which is always unstable. The staggered double row is likewise unstable, except when $h / l=\kappa_{\mathrm{c}}=\left(\cosh ^{-1} \sqrt{ } 2\right) / \pi=0.280550$, at which value $\operatorname{Re}(\sigma)=0$.

Recently, owing partly to the availability of large-scale computing resources, the case in which the vortices are of finite size has attracted attention. Saffman \& Szeto (1981) examined the single row of uniform finite-size vortices, where the vorticity is constant inside each vortex and zero outside. The stream function is continuous and has continuous first derivatives, but the second derivatives have simple jumps across the boundaries of the vortices. They calculated steady shapes numerically, and discussed the stability to two-dimensional disturbances by global energy methods, which have the advantage that they are not limited to infinitesimal disturbances. They concluded that superharmonic disturbances are stable when the vortices are not too large, but there exists a critical size at which superharmonic disturbances become unstable. This provides another mechanism for the evolution of a single row which goes under the name of 'tearing' (Moore \& Saffman 1975). The analysis showed that the pairing instability would not be eliminated by effects of finite size, but the qualitative global analysis could not determine whether the growth rate increased or decreased. (Closed-form quantitative solutions for the case of hollow vortices suggested that the effect of size on the pairing instability is small; see also Baker, Saffman \& Sheffield (1976).) Numerical solutions by Pierrehumbert \& Widnall (1981) for the so-called Stuart vortices show similar behaviour (this paper also contains results for three-dimensional disturbances, which are outside the scope of the present work).

The effect of finite core size on the stability of the Kármán vortex street was considered by Domm (1955), but his undisturbed flow was neither an exact solution of the Euler equations nor of the Navier-Stokes equations. As will be emphasized below, the question of the stabilization by finite size is rather delicate, and Domm's conclusion that finite size does not stabilize is of uncertain significance. The first consistent calculation was carried out by Saffman \& Schatzman (1982), who calculated the values of $\sigma$ for superharmonic disturbances to a double row of uniform vortices and the subharmonic pairing-type instabilities for $p=\frac{1}{2}$. They formulated 
the problem exactly in terms of a nonlinear integro-differential equation based on the 'water-bag' method (see Deem \& Zabusky 1978), whose solutions give the steady shapes and whose Frechet derivative gives the linear eigenvalue problem for the $\sigma$. These equations were solved numerically, thereby giving approximate solutions to an exact formulation. It was found that superharmonic disturbances were stable, as can also be predicted by a global argument, and that the pairing instability could be stabilized by finite size for a range of values of the aspect ratio $h / l$ around $\kappa_{\mathrm{c}}$. Saffman \& Schatzman argued on the basis of a symmetry argument that the pairing disturbances were the most unstable, at least for small vortices, and it was therefore not necessary to consider $p \neq \frac{1}{2}$ with regard to the stability to general disturbances. Unfortunately, as will be discussed below, this conclusion is false.

The problem was considered independently by Kida (1982) in a rather different way. He developed a perturbation expansion in the size of the vortices to obtain approximate equations for the motion of the vortex centroids. In order to do this, it is necessary to calculate the deformation of the vortex cores, which respond quasi-steadily to the motion of the centroids on timescale $l^{2} / \Gamma$, and can oscillate freely on the much shorter timescale $1 / \omega=A / \Gamma$, where $A$ is the core area and $\omega$ is the magnitude of the vorticity in the core. A 'coarse-graining' approximation is made which neglects the high frequencies and produces approximate equations for the vortex centroids which contain the finite-size correction terms of order $A^{2} / l^{4}$. The approximate equations were solved exactly for disturbances of arbitrary subharmonic wavenumber $p$. Kida found for the case $p=\frac{1}{2}$ that his results agreed closely with those of Saffman \& Schatzman, despite the claim by the latter authors based on an analysis of their results that a consistent calculation of the finite-area stabilization around $h / l=\kappa_{\mathrm{c}}$ required retention in the dynamical equations of terms of order $A^{4} / l^{8}$. More important, however, was Kida's prediction that the most unstable disturbance does not occur for $p=\frac{1}{2}$ but for a value of $p$ differing by order $A^{2} / l^{4}$, in contradiction to the Saffman \& Schatzman symmetry argument. He did find, however, that the street was still stabilized by finite area.

This conflict led us to reexamine the problem and tackle the case of general $p$. We will present arguments and results to show that Kida's claim that the most unstable disturbances of the Kármán vortex street is for $p \neq \frac{1}{2}$ is indeed correct and that the Saffman \& Schatzman symmetry argument was flawed, at least for this case. (It is correct for the single row.) On the other hand, we shall also discuss the accuracy of the perturbation expansions and the question whether neglecting terms of order $A^{4} / l^{8}$ in the dynamics is consistent. Our conclusions here are that it is valid for determining the effects of finite area on the oscillation frequencies or growth rates of stable or unstable modes, but not for determining the change of stability properties at the critical spacing ratio $\kappa_{\mathrm{c}}$. In fact, our calculations now predict, somewhat surprisingly, that the street is always unstable, at least for small area, except for one particular value of $h / l$ which depends upon the area. In other words, the qualitative stability properties of the point vortex configuration remains true for finite area except that the most unstable disturbance for $h / l$ not equal to the special value is not the pairing instability.

Our numerical method is sufficiently general that many different flows can be examined with little extra work. We can therefore analyse other cases of interest such as the single row, the symmetrical double row, the circular row in which the vortices are at the vertices of a regular polygon (this provides a model of the effect of curvature on a shear layer), as well as the Kármán vortex street. We can also handle the case in which the vortices in the street have different sizes in the two rows. It is, however, 
shown in Appendix A that the circulations of the vortices must be equal and opposite, so that steady motion of a composite wake-mixing-layer type flow in which $U_{2} \neq U_{1}$ or $U_{2} \neq-U_{1}$ is not possible, as is also suggested by the numerical calculations of Boldman, Brinich \& Goldstein (1976). We shall, however, limit the present paper to the staggered double row, and present results for the other cases in subsequent papers.

After receiving a preprint of the present paper, Kida (private communication) has informed us that there are errors in the formulae (3.10)-(3.17) of Kida (1982). In Kida's notation, these formulae should be

$$
\begin{gathered}
A_{1}=A_{2}=s(-2 E G+2 I J), \\
B_{1}=-s\left(E^{2}+G^{2}+I^{2}-F H\right), \\
B_{2}=-s\left(E^{2}+G^{2}+I^{2}+J^{2}+F H\right), \\
C_{1}=C_{2}=-s F K, \\
D_{1}=s(2 E I-F L+2 G J), \\
D_{2}=s(2 E I+F L+2 G J) .
\end{gathered}
$$

where $s=P^{2} / 2 \pi^{2}=A^{2} / 2 \pi^{2} l^{4}$ in our notation. Further, the equation for the frequencies when found by evaluating exactly the determinant of the coefficient matrix given by Kida's (3.8), i.e retaining all terms of order $A^{4}$, is then

$$
\begin{aligned}
\left(A \pm C_{0}\right)^{2} & +\left(\Lambda \pm C_{0}\right)\left[A_{1}+A_{2} \pm\left(C_{1}+C_{2}\right)\right] \\
& +\left[B_{0}+B_{1} \pm\left(D_{0}+D_{1}\right)\right]\left[B_{0}+B_{2} \mp\left(D_{0}+D_{2}\right)\right]+\left(A_{1} \pm C_{1}\right)\left(A_{2} \pm C_{2}\right)=0
\end{aligned}
$$

instead of Kida's (3.29). When we refer below to analytical results of Kida, this new equation is implied.

It needs to be emphasized that the modified result is not obviously consistent to order $A^{4}$, because terms in the coefficients of the matrix (3.8) are only calculated to order $A^{2}$. However, Kida does state (private communication) that it can be shown that terms of order $A^{4}$ in the coefficient matrix are not important. This statement is consistent with the agreement between our present numerical results and Kida's modified analysis.

\section{Consequences of symmetry}

We now examine some of the general properties that can be established independently of the details of the flow and follow from the structure of the Euler equations and symmetry of the flow.

Because (1.1) is real, it follows that, if $\Psi^{\prime}$ is a mode, so is its complex conjugate $\Psi^{\prime *}$. Thus if $\sigma$ is an eigenvalue, for a value $p, \sigma^{*}$ is an eigenvalue for $-p$ or equivalently $1-p$, and we have the eigenvalue-eigenvector sets

$$
\begin{gathered}
\sigma,\left\{\phi_{n}(y)\right\}, p, \\
\sigma^{*},\left\{\phi_{-n}^{*}(y)\right\},-p .
\end{gathered}
$$

Let us suppose further that the undisturbed flow has fore-and-aft symmetry, i.e. it is possible to choose the origin so that

$$
\Psi(x, y)=\Psi(-x, y)
$$

Then the perturbation equation (1.2) is invariant under the transformation $t \rightarrow-t, x \rightarrow-x$; and we therefore have an eigenvalue-eigenvector set

$$
-\sigma,\left\{\phi_{-n}(y)\right\},-p \text {. }
$$


Taking the complex conjugate of (2.4) gives the set

$$
-\sigma^{*},\left\{\phi_{n}^{*}(y)\right\}, p .
$$

We conclude therefore that for undisturbed flows with fore-and-aft symmetry, and this will include all the cases considered in the present paper, the eigenvalues for given $p$ will come in pairs with $\sigma$ or $-\sigma^{*}$ being an eigenvalue if the other is. It follows that the flow is unstable if $\sigma$ is not pure imaginary. We also conclude that there is a symmetry about $p=0$ or $p=\frac{1}{2}$, since the eigenvalue for $p$ is the complex conjugate of the one for $-p$ or $1-p$. Thus if $\sigma(p)$ denotes the eigenvalue, unstable modes satisfy

$$
\operatorname{Re} \sigma(p)=\operatorname{Re} \sigma(-p) .
$$

There are also damped modes with growth rate $-\operatorname{Re} \phi$ satisfying the same equation. It must be realized, however, that $\sigma$ may be multivalued and the equality in (2.6) may refer to different branches. It was the failure to realize this possibility that led Saffman \& Schatzman to an incorrect assertion about the most unstable modes of the Kármán vortex street.

When symmetry about the axis of the motion exists it gives information on the parity of the components of the eigenvectors but does not appear to give any further results about the eigenvalues themselves. Reflectional symmetry implies that $-\Psi(x+d,-y)$ describes the same flow field, where $d=0$ for the single row or symmetrical double row, and $d=\frac{1}{2}$ for the staggered double row. The perturbation equation is invariant under the transformation $x \rightarrow x+d, y \rightarrow-y, \Psi \rightarrow-\Psi$, and hence there is an eigenvalue-eigenvector set

$$
\sigma,\left\{\mathrm{e}^{2 \pi i n d / l} \phi_{n}(-y)\right\}, p
$$

For $d=0$ the eigenvectors are therefore either even or odd in $y$, and for $d=\frac{1}{2}$ they have a more complex symmetry.

When the vortices are small, the modes of oscillation will correspond to those of an array of small circular vortices, and we expect therefore that the disturbances will be of two types. First will be the cooperative modes of arrays of point vortices, for which the characteristic frequencies are of order $\Gamma / l^{2}$, and second will be the oscillations of a single vortex, for which the characteristic frequencies are $\frac{1}{2} m \omega$, where $\omega=\Gamma / A$ is the magnitude of the vorticity inside the vortex of circulation $\Gamma$ and area $A$, and $m$ is the angular wavenumber. These modes are readily distinguishable, at least for small vortices, and provide a convenient way to group the normal modes.

For the single row (mixing layer), the small-vortex case, analysed by the method described in the preceding paragraph, indicates that there are two degrees of freedom for each type of mode, and the eigenvalues for each $p$ will come in pairs, $\sigma_{1}$ and $\sigma_{2}$, where $\sigma_{2}=-\sigma_{1}^{*}$ if the mode is unstable, and $\sigma_{1}$ and $\sigma_{2}$ are pure imaginary but unequal if the mode is stable. The symmetry about $p=\frac{1}{2}$ also gives a further two eigenvalues $\sigma_{1}^{*}$ and $\sigma_{2}^{*}$ for $1-p$. If unstable, there are then two distinct branches, one with positive and the other with negative real part, which are not equal at $p=\frac{1}{2}$, and analyticity in $p$ and (2.6) then implies that the growth rate of unstable modes is such that

$$
\frac{\mathrm{d} \operatorname{Re} \sigma}{\mathrm{d} p}=0 \text { for } p=\frac{1}{2}
$$

and that $|\operatorname{Re} \sigma|$ is a maximum for the pairing mode. (It could in principle be a minimum but continuous dependence upon the size rules this out for small vortices, since it is a maximum for point vortices.) Also $\operatorname{Im} \sigma$ must be zero at $p=\frac{1}{2}$ for the unstable modes, as otherwise the eigenvalue will not be continuous. Note that $p=0$ 

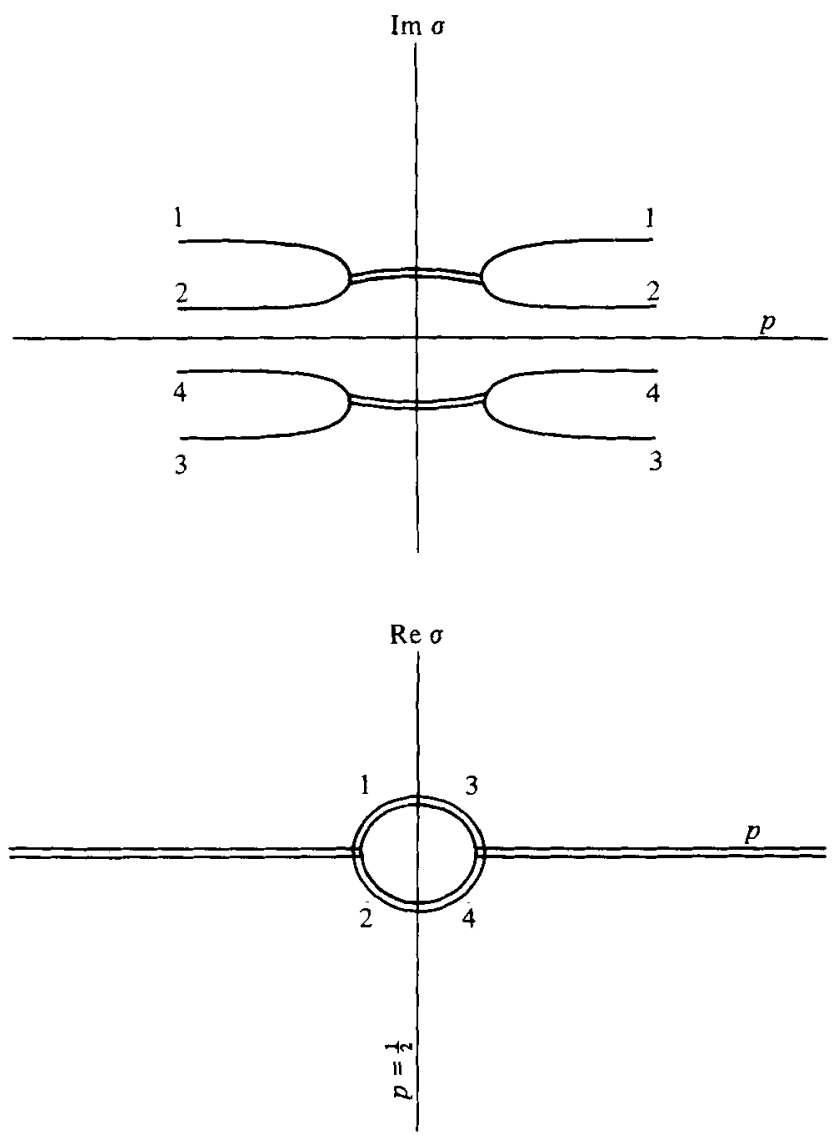

Figure 1. Sketch of dependence of real and imaginary parts of the growth rate on wavenumber $p$ for zero area and a typical value of $\kappa$. For $\kappa=\kappa_{\mathrm{c}}$ the oval shrinks to a point and the imaginary parts form two crosses.

is a singular limit as the disturbance wavelength is going to infinity, and hence (2.8) need not hold there.

In contrast, for the double row (wake), the small-vortex analysis indicates that there are four degrees of freedom for each mode (each row, in effect, contributes two degrees of freedom to the system), and the eigenvalues for each $p$ will come in quartets $\sigma_{1}, \sigma_{2}, \sigma_{3}, \sigma_{4}$, where if unstable $\sigma_{2}=-\sigma_{1}^{*}$ and/or $\sigma_{4}=-\sigma_{3}^{*}$. There are now four branches. The symmetry about $p=0$ or $p=\frac{1}{2}$ now implies that $\sigma_{1}^{*}(p)=\sigma_{3}(-p)$, etc. $\left(\sigma_{1}^{*}(p)=\sigma_{2}(-p)\right.$ violates the continuity of $\operatorname{Im} \sigma$ at $p=\frac{1}{2}$ unless the imaginary part vanishes, and this is contradicted by the small-vortex results.) It does not follow therefore that $\operatorname{Re} \sigma_{1}$ is symmetrical about $p=0$ or $p=\frac{1}{2}$ when there is instability. For $p=\frac{1}{2}$ the quartet of eigenvalues will be such that $\sigma, \sigma^{*},-\sigma$, and $-\sigma^{*}$ are all eigenvalues, but the real and imaginary parts that coincide come from different branches, and (2.8) does not necessarily hold. Contrary to the statement by Saffman $\&$ Schatzman, and in agreement with Kida's results, the $p=\frac{1}{2}$ disturbance will not be the most unstable disturbance.

In figure 1 we show a sketch of the dependence of the four complex growth rates on $p$ for the cooperative modes for zero area and general $\kappa$. In this special case the system is degenerate and there is symmetry about both $\sigma=0$ and $p=\frac{1}{2}$. In addition, 

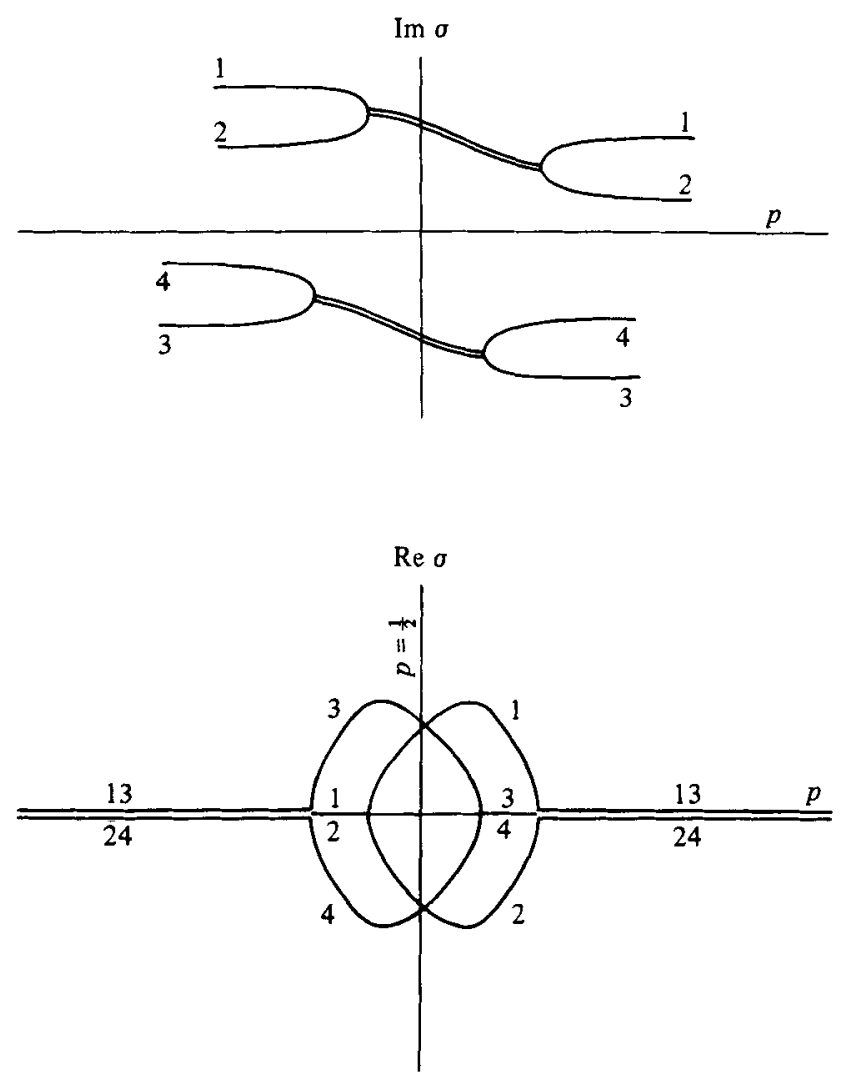

FiquRE 2. Sketch of dependence on $p$ of an unstable quartet of eigenvalues for fixed area and aspect ratio. The pairing mode is unstable, but is not the most-unstable disturbance. Note the form of the symmetry.

for the exceptional value $h / l=\kappa=\kappa_{\mathbf{c}}$, the circle of instability reduces to a point and the imaginary values of $\sigma$ meet in a cross. Figures $2-4$ show how these curves may change when the area of the vortices is finite. Figure 2 is an example where the street remains unstable for $p=\frac{1}{2}$, but the pairing instability is not the most unstable disturbance. Figure 3 is a sketch of a possible behaviour in which the street is unstable, but the pairing instability with $p=\frac{1}{2}$ is stabilized by finite area. Figure 4 is the case where finite area has stabilized the street. It can be seen that the symmetry of reflection about $\sigma=0$ and $p=\frac{1}{2}$ is satisfied, but the symmetry about the axes, which is true for zero area, breaks down when the area is finite.

For $\kappa$ away from $\kappa_{\mathrm{c}}$ it is clear that figure 1 will first change into figure 2 when the area becomes finite, and may later change into figures 3 and 4 when the area becomes larger. Note that these results depend upon fore-and-aft symmetry, but the sizes of the vortices in one row need not be the same as those in the other row. The task is to determine whether the special case of figure 1 for $\kappa=\kappa_{\mathrm{c}}$ ehanges into figure 2, 3 or 4 when $\alpha=A / l^{2}$ is finite for values of $\kappa$ very close to $\kappa_{\mathrm{c}}$. There is also a fourth possibility that for finite area there is an exceptional value of $\kappa$, which should depend upon area, such that figure 3 is degenerate, i.e the ovals shrink into points and the imaginary values meet in crosses.

The high-frequency shape modes, which do not exist for point vortices, are found 

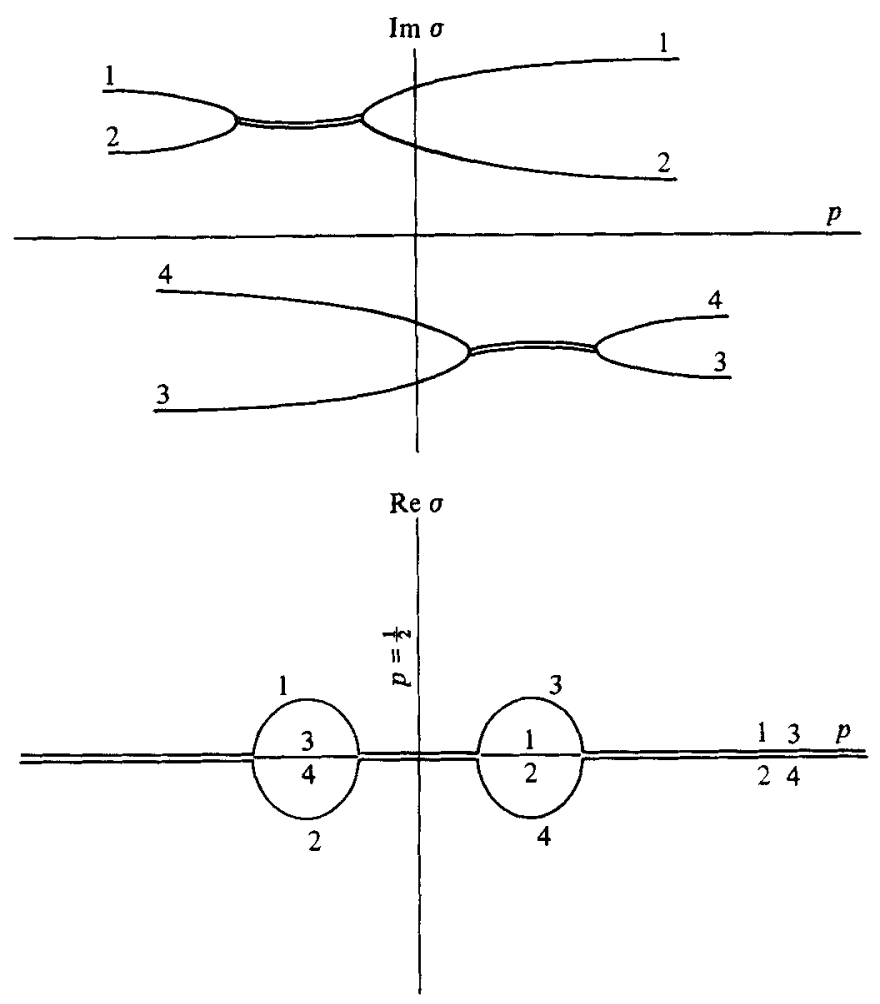

FIGURE 3. Sketch of dependence on $p$ of an unstable quartet of eigenvalues when the pairing mode is stable. A degenerate case may exist in which the ovals shrink to two points and the imaginary parts form two crosses.

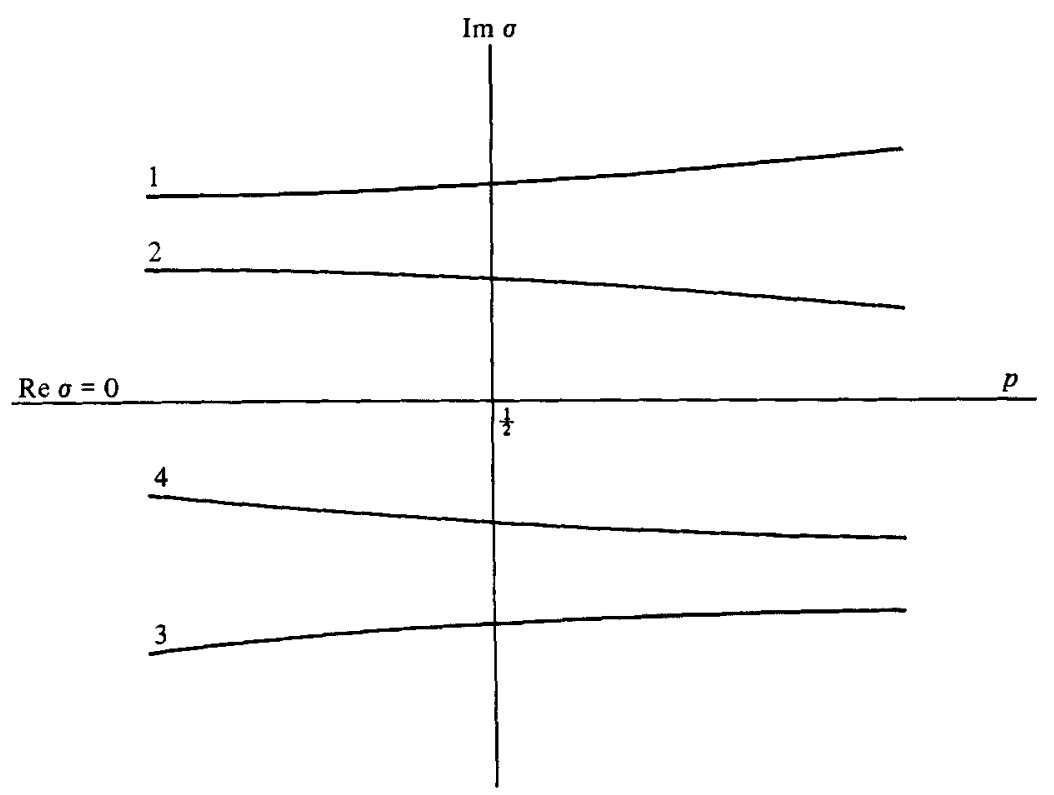

Figure 4. Sketch of dependence of $\sigma$ on $p$ when stabilization by finite area occurs. 
to be stable, provided that the vortices are not too large, and their oscillation frequencies, which will be modified by the interactions, are as in figure 4 .

We now concentrate on the staggered double row, and discuss the detailed problem of what should be calculated and how we shall proceed.

\section{The Kármán street of small vortices}

The complex growth rates $\sigma$ will be the eigenvalues of an infinite system

$$
M \phi=\sigma \phi,
$$

where $\boldsymbol{M}$ will have an expansion of the form

$$
\frac{\Gamma}{l^{2}}\left[\frac{l^{2}}{A} \boldsymbol{M}_{-1}+\boldsymbol{M}_{0}+\frac{A}{l^{2}} \boldsymbol{M}_{1}+\frac{A^{2}}{l^{4}} \boldsymbol{M}_{2}+\ldots\right]
$$

Invariance with respect to the sign of $l$ requires that the expansion of $\boldsymbol{M}$ goes in powers of $\alpha$ and not in powers of $\alpha^{\frac{1}{2}}$, although the detailed solution is an expansion in $1 / l$. The matrices $\boldsymbol{M}_{i}$ depend upon the aspect ratio $\kappa$ and the subharmonic wavenumber $p$. The matrix $\boldsymbol{M}_{-1}$, which produces shape-deformation modes, is given by the simple calculation for the oscillations of an isolated circular vortex, while $\boldsymbol{M}_{\mathbf{0}}$, which produces the cooperative oscillations of the centroids, is found from the Kármán calculation of point vortices. $\boldsymbol{M}_{1}$ etc. have to be found by laborious algebra. The eigenvectors of $\boldsymbol{M}_{-1}$ and $\boldsymbol{M}_{\mathbf{0}}$ are independent. In the absence of degeneracy, which occurs when two or more of the eigenvalues are equal, the eigenvalues will be analytic functions of the parameters.

From the general symmetry arguments of $\$ 2$, we know that the eigenvalues will appear in quartets, which will satisfy quadratic equations

$$
\begin{aligned}
& {\left[\sigma-i \frac{\Gamma}{l^{2}} S_{1}(p, \kappa, \alpha)\right]^{2}=\frac{\Gamma^{2}}{l^{4}} H_{1}(p, \kappa, \alpha),} \\
& {\left[\sigma-i \frac{\Gamma}{l^{2}} S_{3}(p, \kappa, \alpha)\right]^{2}=\frac{\Gamma^{2}}{l^{4}} H_{3}(p, \kappa, \alpha),}
\end{aligned}
$$

where $S_{1}, S_{3}, H_{1}$ and $H_{3}$ are real and dimensionless. The symmetry about $p=0$ or $p=\frac{1}{2}$ implies

$$
S_{3}(p, \kappa, \alpha)=-S_{1}(-p, \kappa, \alpha), \quad H_{3}(p, \kappa, \alpha)=H_{1}(-p, \kappa, \alpha) .
$$

Non-trivial degeneracy occurs if and when $H_{1}$ and $H_{3}$ vanish, and changes of stability, where $\sigma$ changes from complex to pure imaginary, can only occur at such points. With regard therefore to the main problem of the stabilization of the Kármán vortex street by finite-area vortices, the interest is in the functions $H_{1}$ and $H_{3}$ and their zeros. (It is shown in Appendix A that a necessary condition for a steady street is that the circulation of the vertices in the different rows are equal and opposite. The areas can be unequal, but the symmetry arguments leading to (3.3) and (3.4) remain valid. Calculations for the case of different area are currently in progress.)

These functions are known in the limit $\alpha=0$, which can be thought of as either finite-size vortices infinitely far apart, as is appropriate for the shape modes, or point vortices separated by a finite distance, as is appropriate for the cooperative modes. It a ppears from our present numerical calculations and those of Saffman \& Schatzman that $H_{1}$ and $H_{3}$ are negative for the shape modes when $\alpha>0$ (but not too large), which 
therefore remain stable, and we shall therefore concentrate on the cooperative modes. For these,

$$
\begin{aligned}
H_{1}(p, \kappa, 0) & =H_{3}(p, \kappa, 0) \\
& =\frac{1}{4} \pi^{2}\left[2 p(1-p)-\operatorname{sech}^{2} \pi \kappa\right]^{2} \\
& \quad-\frac{1}{4} \frac{\pi^{2}[\cosh 2 \pi \kappa p-2 p \cosh \pi \kappa \cosh (\pi \kappa(1-2 p))]^{2}}{\cosh ^{4} \pi \kappa} .
\end{aligned}
$$

The equality of $H_{1}$ and $H_{3}$ when $\alpha=0$ means that for point vortices the eigenvalues oceur in pairs such that $-\sigma$ is an eigenvalue if $\sigma$ is. This is also a consequence of the fact that point vortices form a Hamiltonian system with $x$ - and $y$-coordinates as conjugate variables. It is important to realize that this additional symmetry is a consequence of a zero-area degeneracy and is broken at finite area.

The $(p, \kappa)$-plane for $\alpha=0$ is divided up into regions of stability and instability, and the boundaries look like a diagonal cross with centre at $p=\frac{1}{2}$ and $\kappa=\kappa_{\mathrm{c}}$, where $\cosh \pi \kappa_{\mathrm{c}}=\sqrt{ } 2$ (see Saffman \& Schatzman 1982, figure 2). For $\kappa$ not close to $\kappa_{\mathrm{c}}$ the effect of finite area is to displace the boundaries of the stability regions, and the results are not of pressing importance. For small area the main interest lies in the behaviour of $p$ close to $\frac{1}{2}$ and $\kappa$ nearly equal to $\kappa_{\mathrm{c}}$. Let

$$
p^{\prime}=p-\frac{1}{2}, \quad \kappa^{\prime}=\kappa-\kappa_{\mathbf{c}},
$$

then we need the expansion of $H_{1}$ and $H_{3}$ for small $p^{\prime}, \kappa^{\prime}$ and $\alpha$.

The numerical results of Saffman \& Schatzman for $p^{\prime}=0$, our present arguments (see also the last paragraph of $\S 4$ ), the analysis of Kida and the numerical results of the present paper indicate that there are no terms in the expansion linear in $\alpha$, and that the dominant terms of the expansion are

$$
H_{1}(p, \kappa, \alpha)=c_{1} \kappa^{\prime 2}+c_{2} p^{\prime 2}+c_{3} \alpha^{2} \kappa^{\prime} \pm c_{4} \alpha^{2} p^{\prime}+c_{5} \alpha^{4}+\ldots
$$

The first coefficient $c_{1}$ is positive, and the second one $c_{2}$ is negative. Both are given analytically by the point-vortex theory. The third and fourth are given analytically by the Kida expansion. The last coefficient $c_{5}$ can be deduced from the Saffman \& Schatzman calculation, which demonstrated that it was negative (and also gives $c_{3}$ ). These numerical results exclude an $\alpha^{3}$ term. An $\alpha^{3} p^{\prime}$ or $\alpha^{3} \kappa^{\prime}$ term could be present, but is unimportant compared with the $\alpha^{4}$ term in its effect on the stability boundary. Notice that Kida's expansion does not include all $\alpha^{4}$ terms consistently, since his calculation of the dynamics neglects terms of order $\alpha^{4}$, and hence his expression for the fifth term is incomplete. (As mentioned earlier, Kida (private communication) claims that the neglected terms are unimportant.)

The numerical values of these coefficients determined by the earlier work are as follows. From the Kármán expressions,

$$
c_{1}=12.1761, \quad c_{2}=-0.700541 .
$$

From the results for the stability boundary for $p=\frac{1}{2}$ given in equation (3.19) of Saffman \& Schatzman, which relate the values of $\alpha$ and $\kappa$ for which $H_{1}$ or $H_{3}$ vanish when $p^{\prime}=0$, we have the estimates

$$
c_{3}^{\mathrm{S}}=-12.88, \quad c_{5}^{\mathrm{s}}=-11.60 .
$$

From the analytical expression given by Kida's equation (3.29) (modified as described at the end of $\$ 1$ ), we find that

$$
c_{3}^{\mathrm{K}}=-13.5243, \quad c_{4}^{\mathrm{K}}=6.48793, \quad c_{5}^{\mathrm{K}}=-11.2663 .
$$


It can be seen that the $c_{3}$ and $c_{5}$ are in reasonable agreement. This is consistent with Kida's statement that his results agree with those of Saffman \& Schatzman for $p=\frac{1}{2}$, as will be discussed in more detail at the end of the section, see (3.20)-(3.22).

The stabilization of the street is determined for small area by (3.8). We complete the squares and write (3.8) as

$$
H_{\frac{1}{3}}=c_{1}\left(\kappa^{\prime}+\frac{c_{3}^{2} \alpha^{2}}{2 c_{1}}\right)+c_{2}\left(p^{\prime} \pm \frac{c_{4}^{2} \alpha^{2}}{2 c_{2}}\right)+\alpha^{4}\left(c_{5}-\frac{c_{3}^{2}}{4 c_{1}}-\frac{c_{4}^{2}}{4 c_{2}}\right)
$$

The stabilization or destabilization of the street by finite area therefore depends upon the sign of

$$
D=c_{5}-\frac{c_{3}^{2}}{4 c_{1}}-\frac{c_{4}^{2}}{4 c_{2}}
$$

If $D$ is positive the street is made unstable by finite area in the vicinity of the zero-area point of stability. If $D$ is negative the street is stabilized for a range of $\kappa$ of order $\alpha^{2}$ by finite area.

If $D=0$ then there remains an isolated point of stability in the $(k, p)$-plane, but the aspect ratio of the stable street is altered by a value of order $\alpha^{2}$. In this case it would be necessary to go to yet higher order in $\alpha$ to determine the stability question.

For a fixed $\alpha$, the stability boundaries in the $\left(\kappa^{\prime}, p^{\prime}\right)$-plane are given by the vanishing of (3.12), which gives two hyperbolae. The interiors of the hyperbolae, marked by cross-hatching, contain the unstable modes. There are two main possibilities depending upon the values of the $c$-coefficients. Figure 5 shows the stability boundaries for $p$ close to $\frac{1}{2}$ and $\kappa$ near $\kappa_{\mathrm{c}}$ when $D$ is negative. There is a region of stability bounded by the two hyperbolae with centres at $\kappa^{\prime}=\lambda \alpha^{2}$ and $p^{\prime}= \pm \mu \alpha^{2}$, where

$$
\lambda=\frac{-c_{3}}{2 c_{1}}, \quad \mu=\frac{-c_{4}}{2 c_{2}} .
$$

The distance between the vertices giving the range of $\kappa$ for which there is stability is

$$
\delta \kappa=\left(-D / c_{1}\right)^{\frac{1}{2}} \alpha^{2} .
$$

In this case, finite area stabilizes the street for a range of aspect ratio.

On the other hand, if $D$ is positive, then the stability boundaries are as shown in figure 6. Now, there is always a value of $p$ which gives an unstable mode and finite area destabilizes the street by removing the neutral case. If

$$
\mu>\left(-D / c_{2}\right)^{\frac{1}{2}}
$$

there is a lens of stability along the $p^{\prime}=0$ axis, and the boundaries are as shown in figure 6 . The pairing mode is then stabilized, but there is a range of unstable values of $p$ of width

$$
\delta p=\left(-D / c_{2}\right)^{\frac{1}{2}} \alpha^{2} .
$$

If (3.16) is not satisfied the hyperbolae overlap and there is no stabilization of the pairing mode.

If $D=0$ the hyperbolae degenerate into crosses meeting at the points given by (3.14), and the street is stable for one special value of $\kappa$ equal to $\kappa_{\mathrm{c}}+\lambda \alpha^{2}$.

Using Kida's modified values given by (3.11), we find that $D=0$. Using Saffman $\&$ Schatzman's estimate of $c_{3}$ and $c_{5}$, and Kida's value of $c_{4}$, we have $D=0.037$. It appears therefore that $D$ is small and possibly zero, and the question of stabilization by finite area is delicate. Either a consistent perturbation expansion of order $\alpha^{4}$ should 


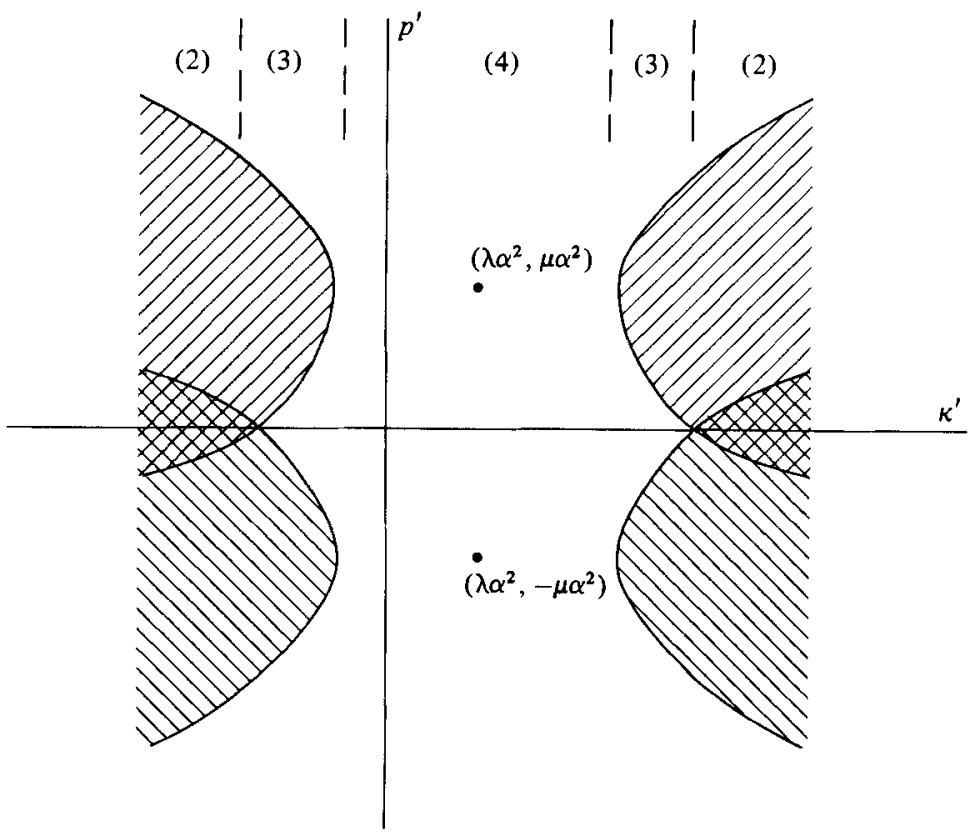

Figure 5. Stability boundary in the $\left(k^{\prime}, p^{\prime}\right)$-plane when finite area stabilizes. Hyperbolae would cross at centres if $D=0$. Cross-hatched areas denote instability regions for modes 1 and 3 . Numbers in parentheses show corresponding figure for growth rate as function of $p$.

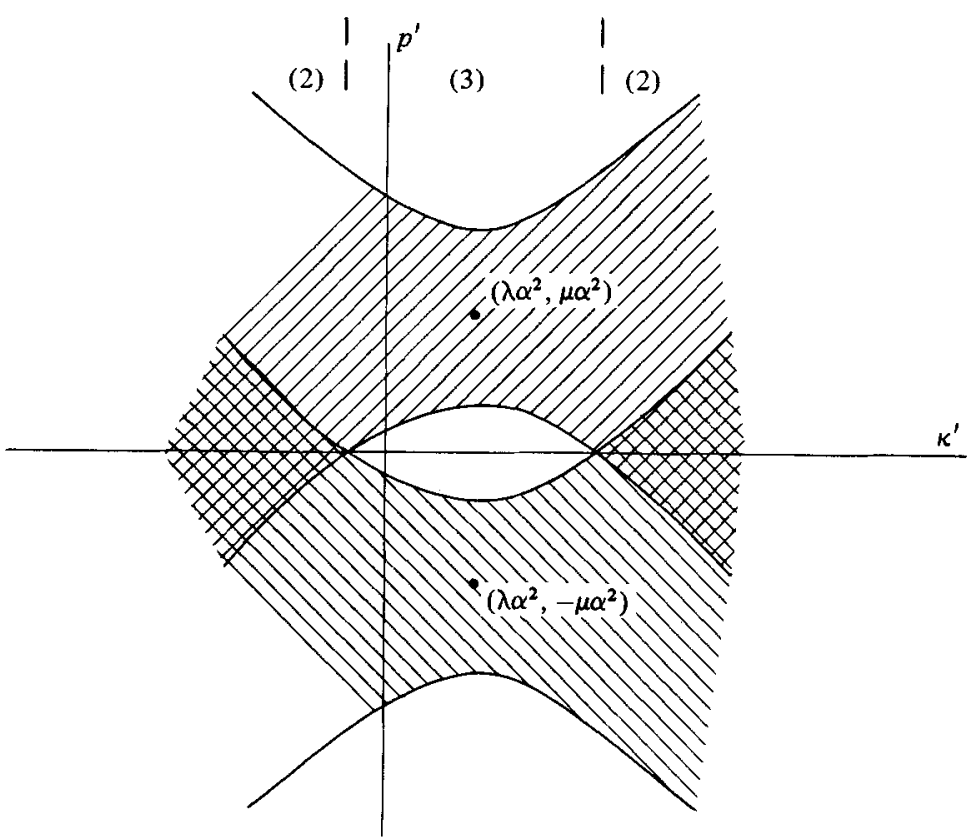

Figure 6. Stability boundary in the $\left(\kappa^{\prime}, p^{\prime}\right)$-plane when finite area destabilizes. In the case shown the pairing instability $p^{\prime}=0$ is stabilized. 
be carried out, or a further numerical investigation be attempted. The algebra entailed in the former is heavy, although Kida now claims to have done it. Saffman \& Schatzman did consider carrying out such an expansion for the simpler $p=\frac{1}{2}$ case, but decided in favour of a numerical calculaton, and it was again decided when the present work was started in 1982 to go to a numerical approach.

We discuss in $\$ \S 4$ and 5 our method for calculating the steady shapes and stability boundaries, which we use to investigate the stability of the cooperative modes. It also gives the high-frequency shape modes, and has the advantage that it is easily applied to a wider class of problems. With respect to the street stability, the numerical results indicate that $D$ is actually zero to within the limit of numerical accuracy. Kida's modified results agree with ours. However, as will be discussed later, our results appear to show that $D=0$ for all $\alpha$, not just to order $\alpha^{4}$.

Kida's expansion gives the coefficient of $\alpha^{2}$ exactly in closed form for all $p$ and $\kappa$. Away from the critical point, there will be values $p_{\mathrm{s}}$ and $\kappa_{\mathrm{s}}$ at which the stability of point vortices changes. To determine the effects of finite area for these values, we require an expansion

$$
H_{1}(p, \kappa, \alpha)=c_{1}\left(\kappa-\kappa_{\mathrm{s}}\right)+c_{2}\left(p-p_{\mathrm{s}}\right)+c_{3} \alpha^{2},
$$

and a similar expansion for $H_{3}$. Here $c_{1}$ and $c_{2}$ are known functions of $p_{\mathrm{s}}$ which vanish for $p_{\mathrm{s}}=\frac{1}{2}$, and the same is true for $c_{3}$, which is given by Kida's results. For $p_{\mathrm{s}} \neq \frac{1}{2}$, (3.18) is sufficient for the determination of the stability properties for small $\alpha$. However, the coefficients in (3.18) vanish when $p_{\mathrm{s}}=\frac{1}{2}$, and there the expansion (3.8) is required.

The discussion of this section shows that from Kida's expressions for $H_{1}$ and $H_{3}$ correct to $O\left(\alpha^{2}\right)$ combined with the numerical or modified Kida results which give the coefficient $c_{5}$, we can construct an expression for the effect of finite size on the stability boundaries which is uniformly valid to $O\left(\alpha^{2}\right)$ for all $p$ and $\kappa$. We have for the cooperative modes

$$
\begin{aligned}
H_{1}(p, \kappa, \alpha)= & \text { 'linear theory' } \\
& + \text { 'Kida' } \alpha^{2} \\
& + \text { 'numerical or modified Kida' } \alpha^{4},
\end{aligned}
$$

with $H_{3}$ given by (3.5). Kida's analysis also gives $S_{1}$ and $S_{3}$ definitely correct to $O\left(\alpha^{2}\right)$, and probably correct to $O\left(\alpha^{4}\right)$.

For the special case of disturbances with $p=\frac{1}{2}, p^{\prime}=0$, Saffman \& Schatzman found that for small area the street was stabilized for aspect ratios in the range

$$
-0.583 \alpha^{2}<\kappa^{\prime}<1.644 \alpha^{2} .
$$

According to (3.8) the range of values is

where

$$
\begin{gathered}
-b_{1} \alpha^{2}<\kappa^{\prime}<b_{2} \alpha^{2}, \\
c_{3}=c_{1}\left(b_{1}-b_{2}\right), \quad c_{5}=-c_{1} b_{1} b_{2} .
\end{gathered}
$$

Kida's values of $c_{3}$ and $c_{5}$ give $b_{1}=0.55, b_{2}=1.67$; which agree reasonably with the values found by Saffman \& Schatzman.

\section{Calculation of steady shapes}

We describe the method here in the context of the staggered double row. Modifications for other flow configurations are simple and easy to implement. Saffman \& Schatzman employed a boundary-integral method. This has the advantage that 
it can be employed for finite area, but it suffers from the disadvantage of being rather hard to implement for disturbances of arbitrary wavenumber (although this possibility is under investigation) and also being hard to check analytically. The method we employ here follows one suggested by Dr Javier Jimenez, and is based on an expansion in inverse powers of $l$. This can be thought of as an expansion in core radius or $\alpha^{\frac{1}{2}}$, but we choose to keep the area of the vortices finite and suppose the separation $l$ to be large, with $h / l$ kept fixed. In principle, the algebra can be done and a consistent perturbation expansion developed, but this is beyond our present resources (even with the aid of symbolic-manipulation programs), and we do the algebra arithmetically. Unfortunately, the detailed approach does not allow the retention of high-order terms in a completely uniform manner, so checking with Kida's perturbation results suffers from uncertainty.

The shape of a vortex in the first row is assumed to be described by the exterior conformal map of the unit circle $|\zeta|=1$ :

$$
z=R_{1} \zeta\left\{1+\frac{a_{1}}{\zeta}+\frac{a_{2}}{\zeta^{2}}+\ldots\right\}
$$

Vortices in the second row have the expansion

$$
z=R_{2} \zeta\left\{1+\frac{b_{1}}{\zeta}+\frac{b_{2}}{\zeta^{2}}+\ldots\right\}
$$

These series may fail to converge before the solutions actually break down by vortices coming into contact, but we assume that there is convergence for a finite range of area.

Our first task is to calculate the velocity field induced by a single vortex containing vorticity $\omega_{1}$ of shape (4.1). To do this, we employ (following Jimenez) the so-called Schwarz functions, which are analytic functions of $z$ equal to $z^{*}$ on a contour. To find the Schwarz function for the contour (4.1), we expand the expression conjugate to (4.1), remembering that $\zeta^{*}=1 / \zeta$ on the contour,

$$
z^{*}=R_{1}^{*} / \zeta\left\{1+a_{1}^{*} \zeta+a_{2}^{*} \zeta^{2}+\ldots\right\}
$$

as a Laurent series in $z$, giving an expansion

$$
z^{*}=\frac{g_{0}}{z}+\frac{g_{1}}{z^{2}}+\frac{g_{2}}{z^{3}}+\ldots+f_{0}+f_{1} z+f_{2} z^{2}+\ldots
$$

The complex velocity $u-\mathrm{i} v$ induced by the vortex is analytic outside the vortex, and such that $u-\mathrm{i} v+\frac{1}{2} \mathrm{i} \omega_{1} z^{*}$ is analytic inside the vortex. It follows by inspection that the exterior velocity field is

$$
u-\mathrm{i} v=\frac{-\mathrm{i} \omega_{1}}{2}\left\{\frac{g_{0}}{z}+\frac{g_{1}}{z^{2}}+\frac{g_{2}}{z^{3}}+\ldots\right\} .
$$

The interior velocity field, which can be expressed in terms of the $f_{n}$ etc., is not required. It remains to describe how the $g_{n}$ are found in terms of the $a_{n}$.

To do this, we multiply (4.4) by $z^{n}$ and integrate around a contour in the $z$-plane, giving

$$
\begin{aligned}
g_{n} & =\frac{1}{2 \pi \mathrm{i}} \oint z^{*} z^{n} \mathrm{~d} z=\frac{1}{2 \pi \mathrm{i}} \oint z^{*} z^{n} \frac{\mathrm{d} z}{\mathrm{~d} \zeta} \mathrm{d} \zeta \\
& =\text { coefficient of } \zeta^{-1} \text { in product } z^{*} z^{n} \frac{\mathrm{d} z}{\mathrm{~d} \zeta}
\end{aligned}
$$

when (4.1) is substituted for $z$ and (4.3) for $z^{*}$. 
Similarly

$$
g_{n}^{*}=- \text { coefficient of } \zeta^{-1} \text { in product } z z^{* n} \frac{\mathrm{d} z^{*}}{\mathrm{~d} \zeta}
$$

the minus sign arising from reversal of the sense of the contour.

There are alternative expressions for the $g_{n}$ in terms of moments of the shape of the vortex. Since

$$
u-\mathrm{i} v=\frac{-\mathrm{i}}{2 \pi} \omega_{1} \iint_{1} \frac{\mathrm{d} x^{\prime} \mathrm{d} y^{\prime}}{z-z^{\prime}}
$$

it follows that

$$
\pi g_{n}=\iint_{1} z^{\prime n} \mathrm{~d} x^{\prime} \mathrm{d} y^{\prime}
$$

where the integral is over the vortex. In particular, $g_{0}$ is the area/ $\pi$, and $g_{1}$ is proportional to the displacement of the centroid relative to the $z$-origin.

Similarly, we obtain an expression for velocity induced by the vortex (4.2):

$$
u-\mathrm{i} v=\frac{-\mathrm{i} \omega_{2}}{2}\left\{\frac{h_{0}}{z}+\frac{h_{1}}{z^{2}}+\frac{h_{2}}{z^{3}}+\ldots\right\},
$$

where the $h_{n}$ are related to the $b_{n}$ by the formula analogous to (4.6).

We also obtain the conjugate velocity

$$
u+\mathrm{i} v=\frac{\mathrm{i} \omega_{1}}{2}\left\{\frac{g_{0}^{*}}{z^{*}}+\frac{g_{1}^{*}}{z^{* 2}}+\frac{g_{2}^{*}}{z^{* 3}}+\ldots\right\},
$$

and similarly for the second vortex, where the $g_{n}^{*}$ are related to the $a_{n}$ and $a_{n}^{*}$ by (4.7).

An expression can now be written down for the complex velocity produced by the two staggered rows. The origins in the first row will be supposed to be at the points $m l,-\infty<m<\infty$, and in the second row at the points $m l-l(d+i \kappa)$. The separation $l$ need not be real; its phase determines the angle between the $x$-axis and the direction of the rows. Indeed, it proved convenient in the actual calculations to take $l$ to be pure imaginary. The relative stagger $d$ and the aspect ratio $\kappa$ are real. For the Kármán vortex street, $d=\frac{1}{2}$. For the symmetrical double row, $d=0$. Arbitrary values of $d$ may be considered, but we shall not do so. Then at a point with coordinate $z$ relative to a vortex in the first row

$$
\begin{aligned}
u-\mathrm{i} v=\frac{-\mathrm{i} \omega_{1}}{2} \sum_{n=0}^{\infty} \sum_{m=-\infty}^{\infty} & \frac{g_{n}}{(z-m l)^{n+1}} \\
& -\frac{\mathrm{i} \omega_{2}}{2} \sum_{n=0}^{\infty} \sum_{m=-\infty}^{\infty} \frac{h_{n}}{(z-m l+l(d+\mathrm{i} \kappa))^{n+1}}+U-\mathrm{i} V,
\end{aligned}
$$

and at a point with coordinate $z$ relative to a vortex in the second row

$$
\begin{aligned}
u-\mathrm{i} v=\frac{-\mathrm{i} \omega_{2}}{2} \sum_{n=0}^{\infty} \sum_{m=-\infty}^{\infty} & \frac{h_{n}}{(z-m l)^{n+1}} \\
& \quad-\frac{\mathrm{i} \omega_{1}}{2} \sum_{n=0}^{\infty} \sum_{m=-\infty}^{\infty} \frac{g_{n}}{(z-m l-l(d+\mathrm{i} \kappa))^{n+1}}+U-\mathrm{i} V .
\end{aligned}
$$

$U$ and $V$ are components of the velocity at infinity which balances the self-induced motion of the street and brings the vortices to rest.

Expressions for the conjugate velocities $u+i v$ are obtained by replacing $i$ with $-i$, $g_{n}$ with $g_{n}^{*}, h_{n}$ with $h_{n}^{*}$, and $l$ with $l^{*}$. 
For steady motion the shapes of the vortices are given by the condition that their boundaries are streamlines, i.e. $u-\mathrm{i} v$ is parallel to the tangent $\mathrm{d} z / \mathrm{d} s$. This is expressed by the equation

$$
R(\zeta) \equiv(u-\mathrm{i} v) \zeta \frac{\mathrm{d} z}{\mathrm{~d} \zeta}+(u+\mathrm{i} v) \zeta \frac{\mathrm{d} z^{*}}{\mathrm{~d} \zeta}=0
$$

when $|\zeta|=1$, to be satisfied on the first and second vortices, i.e. with $u-i v$ given by (4.12) and $z$ given by (4.1), and $u-\mathrm{i} v$ given by (4.13) and $z$ given by (4.2), and the appropriate conjugate expressions, respectively. This provides equations for the unknown coefficients $R_{1}, a_{n}, R_{2}, b_{n}$, and the translation velocity $Q=U-\mathrm{i} V$ and $q^{*}=U+\mathrm{i} V$.

It is expected that locally unique (isolated) solutions will exist if the areas of the vortices, the positions of the centroids, and the strengths of the vortices are given. The question to be discussed now is the actual procedure to be employed.

The straightforward way, and the one that is implicit in the present formulation, is to develop a series expansion in $\alpha$, the solution for $\alpha=0$ being known. It is convenient to suppose that the vortices have finite areas and strengths, and that the limit $\alpha=0$ is obtained by letting $l \rightarrow \infty$. Then we can develop the velocity by expanding the terms in (4.12) and (4.13) and their conjugates as power series in $1 / l$ and calculate the unknowns as series in the same variable. This is feasible in principle, but impossible in practice at present, especially when it is remembered that the stabilization problem requires retention of terms of order $l^{-9}$ in order to retain consistently effects of order $\alpha^{4}$. An alternative approach, and the one that we employed, is to use the computer to do the algebra arithmetically as follows.

We pick two integers $N$ and $L$. The series (4.1), (4.2) and their conjugates are truncated to keep the first $N+1$ terms, i.e. we include $a_{N}, b_{N}, a_{N}^{*}$ and $b_{N}^{*}$. Newton iteration will be employed to find the values of these (in general) complex numbers. For this purpose we then calculate numerically, starting with a first guess for the $2 N+2$ unknowns $R_{1}, a_{1}, \ldots, a_{N}, R_{1}^{*}, a_{1}^{*}, \ldots, a_{N}^{*}$, from (4.6) and (4.7), the values of $g_{0}$, $g_{1}, \ldots, g_{N}, g_{0}^{*}, g_{1}^{*}, \ldots, g_{N}^{*}$. In fact, $g_{0}$ and $g_{0}^{*}$ are put equal to the given area $A_{1}$ of the vortex divided by $\pi$, and the difference between the values calculated from $(4.6)$ and (4.7) are used as a check on the accuracy of the calculation. Similarly, we calculate the $h_{n}$ and $h_{n}^{*}$. For reasons that are important for the stability calculation and will be made clear later, we treat quantities and their conjugates as independent variables, e.g. $R_{1}$ and $R_{1}^{*}$ are regarded as independent complex numbers. Of course, the solution will not be physically sensible unless the final answers are complex conjugates of each other. So far, we then have $4 N+4$ complex unknowns.

We now expand (4.12) as a power series in $1 / l$, retaining terms of order $l^{-L}$. This gives a triple series

$$
\begin{aligned}
u-\mathrm{i} v= & \frac{-\mathrm{i} \omega_{1}}{2} \sum_{n=0}^{N}\left[\frac{g_{n}}{z^{n+1}}+\sum_{p=0}^{L-n-1} g_{n} B(p, n+1) \frac{z^{p}(-1)^{p+n+1}}{l^{p+n+1}} \sum_{\substack{m=-\infty \\
m \neq 0}}^{\infty} \frac{1}{m^{p+n+1}}\right] \\
& -\frac{\mathrm{i} \omega_{2}}{2} \sum_{n=0}^{N} \sum_{p=0}^{L-n-1} g_{n} B(p, n+1) \frac{z^{p}(-1)^{p+n+1}}{l^{p+n+1}} \sum_{m=-\infty}^{\infty} \frac{1}{(m-d-\mathrm{i} \kappa)^{p+n+1}}+Q,
\end{aligned}
$$

where $B(p, n)$ is the coefficient of $z^{p}$ in the binomial expansion of $(1+z)^{-n}$. The vorticities are $\omega_{1}=\Gamma / A_{1}$ and $\omega_{2}=-\Gamma / A_{2}$. The sums with respect to $m$ can be done in closed form; see Appendix B. We now substitute the truncated expansion (4.1) into (4.15) and obtain $u-\mathrm{i} v$ evaluated on the boundary of the vortex as a series of 
positive and negative powers of $\zeta$. Differentiation of (4.1), etc., gives a series in $\zeta$ for $\mathrm{d} z / \mathrm{d} \zeta$, etc., and substitution into (4.14) gives a series of positive and negative powers of $\zeta$ with complex coefficients which are analytical functions of $d$ and $\kappa$, contain inverse powers of $l$, and are numerical functions of the $4 N+4$ unknowns $R_{1}, a_{n}$, etc. They also contain the two unknown complex velocities $Q=U-\mathrm{i} V$ and $Q^{*}=U+\mathrm{i} V$, which will also be regarded as independent complex unknowns.

To obtain equations for these $4 N+6$ unknowns we calculate the coefficients of $\zeta^{n}$ in the expression (4.14) for values of $n$ in the range $-N \leqslant n<N$, for vortices in the first and second row, and denote these $4 N+2$ complex quantities $(2 N+1$ for each row) by $E_{1}(n)$ and $E_{2}(n)$. We obtain $4 N+2$ equations for the unknowns by requiring

$$
E_{1}(n)=0, \quad E_{2}(n)=0 \quad(-N \leqslant n \leqslant N) .
$$

We have not yet said anything about the position of the origins inside the vortices, from which the separation $\kappa$ is measured. It is convenient to require that the centroids coincide with the origins. These constraints upon the variables are equivalent to the four equations

$$
g_{1}=g_{1}^{*}=h_{1}=h_{1}^{*}=0 \text {. }
$$

Two further equations are found from the condition that the vortices have given area. Defining the area by

$$
A_{1}=\pi R_{1} R_{1}^{*}\left\{1-a_{2} a_{2}^{*}-2 a_{3} a_{3}^{*}-\ldots-(N-1) a_{N} a_{N}^{*}\right\},
$$

and a similar expression for $A_{2}$, which are correct when starred quantities are complex conjugates, and which are identical with $g_{0}=g_{0}^{*}=A_{1} / \pi$ in this case, we have two further equations.

Thus altogether we have $4 N+8$ equations for $4 N+6$ unknowns. The equations are, however, not independent. First $E_{1}(0)$ and $E_{2}(0)$ are identically zero, and secondly there are two independent constraints on the $E_{1}(n)$ and $E_{2}(n)$. The reasons are as follows.

On the surface of the vortex

$$
(u-\mathrm{i} v) \mathrm{d} z=\left(u_{s}+\mathrm{i} u_{n}\right) \mathrm{d} s,
$$

where $u_{s}$ and $u_{n}$ are the tangential and normal components and $\mathrm{d} s$ is the element of length. Also $\zeta=\exp (\mathrm{i} \theta)$. It follows that $2 u_{n} \mathrm{~d} s=R(\zeta) \mathrm{d} \theta$. Now the velocity field that we construct above is an exact solution. of the Euler equations, and hence must automatically satisfy the equation of continuity. Thus automatically

$$
\oint u_{n} \mathrm{~d} s=0=\int_{0}^{2 \pi} R(\zeta) \mathrm{d} \theta=2 \pi E(0),
$$

and hence the vanishing of $E_{1}(0)$ and $E_{2}(0)$ are just consistency checks and not independent equations.

Next consider the consistency condition (A 6) derived in Appendix A. Since

$$
\iint(u \pm \mathrm{i} v) \mathrm{d} x \mathrm{~d} y=\oint(x \pm i y) u_{n} \mathrm{~d} s
$$

we can rewrite this equation for constant vorticity as

$$
\omega_{1} \int_{1}\left(z \pm z^{*}\right) R(\zeta) \mathrm{d} \theta+\omega_{2} \int_{2}\left(z \pm z^{*}\right) R(\zeta) \mathrm{d} \theta=0
$$

Thus we have two constraints relating the components of $R(\zeta)$ on the two vortices which are imposed by kinematics. Since $z$ is given by (4.1), etc., the relations are 
between all the $E_{1}(n)$ and $E_{2}(n)$, but, since the dominant terms in the expansions are the leading ones, it is expected that it suffices to throw away the equations for $E_{2}(1)$ and $E_{2}(-1)$. The existence of (4.21) will ensure that the converged solution will make these quantities zero automatically, and in fact the accuracy of the solution can be monitored by their values.

To summarize, we now have $4 N+4$ independent complex equations from (4.17) (4 equations), (4.18) (2 equations), $(4.16 a)$ with $n=0$ excluded ( $2 N$ equations), and (4.16b) with $n=0, n=1$ and $n=-1 \operatorname{excluded}(2 N-2)$ equations). However, we still have $4 N+6$ complex unknowns $R_{1}, a_{n 1}, R_{2}, a_{n 2}$, their conjugates, and the two complex velocities. 'Two further equations are therefore required. These are obtained by noting that the phase of $\zeta$ is arbitrary, and that this can be fixed by speeifying the phases of $R_{1}$ and $R_{2}$. We do this by requiring that in the final solution $R_{1}$ and $R_{2}$ should be real, which is imposed by the two complex equations

$$
R_{1}=R_{1}^{*}, \quad R_{2}=R_{2}^{*} .
$$

The number of equations and unknowns is now equal. The test that the counting has been done consistently is the non-vanishing of the Jacobian of the system. (The constraints embodied in (4.21) were in fact discovered numerically by the vanishing of the Jacobian when the $n= \pm-1$ equations of (4.16b) were not excluded.)

For given values of the parameters, a first guess was taken, usually the circular vortex approximation, and solutions were calculated by solving the system with Newton iteration. It is necessary to decide on suitable values of $N$ and $L$. Taking $L=1$ gives circular vortices, since the induced velocity in the neighbourhood of a vortex is then constant. To calculate deformation consistently to order $\alpha$, it is necessary to take $L=2$, and it is then sufficient to take $N=2$, which gives vortices of elliptical shape. This corresponds exactly to what can be called the elliptical-vortex approximation (Saffman \& Szeto 1981). The exterior velocity induced by a uniform vortex of elliptical shape differs by terms of relative order $\alpha^{2}$ from that of a circular vortex of the same circulation, so at this order the cooperative behaviour, i.e. translation velocity and stability properties, will be the same as for point vortices. This explains why there are no terms linear in $\alpha$ in (3.8). The first significant effect of area comes with $L=5$, which gives a consistent calculation of $O\left(\alpha^{2}\right)$ effects. The value of $N$ should be at least $L$, and there is little point in taking larger values. The accuracy of the solution can be tested by comparing with the results of Saffman \& Schatzman (1982) and the steady solutions correct to order $\alpha^{2}$ given by Kida. Unfortunately, the numerical method does not ignore smaller terms, and results obtained with $L=5$ cannot agree exactly with Kida's, but the difference should be of order $\alpha^{4}$. For the investigation of stability, at least near the critical value of $\kappa=\kappa_{\mathrm{c}}$, it is necessary to take $L=9$ and $N=8$ or larger to ensure retention of all terms $O\left(\alpha^{4}\right)$.

\section{Stability}

We wish to consider the stability to infinitesimal two-dimensional disturbances of the steady shapes calculated by the method described in $\$ 4$. It is assumed that the boundaries are deformed by disturbances and that the vorticity inside the vortices remains constant. There is of course a continuum of oscillations associated with changes of the vorticity distribution, but these are not relevant to the intrinsic stability of the street to disturbances produced by motion of boundaries or the action of conservative forces. We suppose now that the coefficients of the expansions (4.1), etc., are perturbed by infinitesimal functions of time, which are denoted by primes. 
The linearized unsteady boundary condition that the vortex boundaries are material boundaries gives the equation

$$
\frac{\mathrm{d} z^{*}}{\mathrm{~d} t} \zeta \frac{\mathrm{d} z}{\mathrm{~d} \zeta}+\frac{\mathrm{d} z^{\prime}}{\mathrm{d} t} \zeta \frac{\mathrm{d} z^{*}}{\mathrm{~d} \zeta}=\left(u^{\prime}-\mathrm{i} v^{\prime}\right) \zeta \frac{\mathrm{d} z}{\mathrm{~d} \zeta}+\left(u^{\prime}+\mathrm{i} v^{\prime}\right) \zeta \frac{\mathrm{d} z^{*}}{\mathrm{~d} \zeta}+(u-\mathrm{i} v) \zeta \frac{\mathrm{d} z^{\prime}}{\mathrm{d} \zeta}+(u+\mathrm{i} v) \zeta \frac{\mathrm{d} z^{*}}{\mathrm{~d} \zeta}
$$

to be satisfied on each vortex of the rows. Unprimed quantities denote the undisturbed steady state.

We now have to handle the problem that for subharmonic disturbances the oscillations of each vortex may be different, so that we appear to be faced with infinitely many equations for infinitely many unknowns, irrespective of any truncation that is employed. This difficulty is overcome as follows. The velocity induced by a vortex is an analytic function of the coefficients. Let $(u-\mathrm{i} v)_{m}$ denote the velocity induced by the vortex centred at $m l$. Then, for example, the change in this velocity at a fixed value of $z$ produced by the perturbation $a_{n, m}^{\prime}$ is

$$
\left(u^{\prime}-\mathrm{i} v^{\prime}\right)_{m}=\frac{\mathrm{d}(u-\mathrm{i} v)}{\mathrm{d} a_{n}} a_{n, m}^{\prime},
$$

where $u-\mathrm{i} v$ is given by (4.5) as a function of the undisturbed coefficients. Because of the periodicity of the undisturbed motion, the derivative in (5.2) is the same function of $z-m l$ for every vortex in the same row. Expressions similar to (5.2) hold for the conjugate velocity and the velocities induced by the vortices in the other row. It follows therefore that normal-morle solutions of (5.1) can be obtained by restricting attention to one vortex in each row, namely the vortices given by (4.1) and (4.2), and supposing that the perturbations of the other vortices are related to the perturbations of these vortices by relations

$$
\begin{aligned}
& a_{n, m}^{\prime}=a_{n}^{\prime} \mathrm{e}^{\mathrm{i} m f}, \quad a_{n, m}^{\prime *}=a_{n}^{\prime *} \mathrm{e}^{\mathrm{i} m f}, \quad R_{1, m}^{\prime}=R_{1}^{\prime} \mathrm{e}^{\mathrm{i} m f}, \quad R_{1, m}^{\prime *}=R_{1}^{\prime *} \mathrm{e}^{\mathrm{i} m f}, \\
& b_{n, m}^{\prime}=b_{n}^{\prime} \mathrm{e}^{\mathrm{i} m f}, \quad b_{n, m}^{\prime *}=b_{n}^{\prime *} \mathrm{e}^{\mathrm{i} m f}, \quad R_{2, m}^{\prime}=R_{2}^{\prime} \mathrm{e}^{\mathrm{i} m f}, \quad R_{2, m}^{\prime *}=R_{2}^{\prime *} \mathrm{e}^{\mathrm{i} m f},
\end{aligned}
$$

where $f$ is related to the subharmonic wavenumber $p$ by

$$
f=2 \pi p \text {. }
$$

Notice the subtle point that, although the undisturbed solution must have the property that $a_{n}$ and $a_{n}^{*}$, etc., are complex conjugates for it to be physically sensible, this need not be true of infinitesimal perturbations. There is no contradiction here, as will be explained in more detail later. It is of course just the manifestation in the present context of the fact that the eigenfunction of a real problem may be complex.

The perturbed velocity $u^{\prime}-\mathrm{i} v^{\prime}$ can now be calculated. We have the undisturbed velocity induced by the vortices as given by $(4.12)$ :

$$
u-\mathrm{i} v=\frac{-\mathrm{i} \omega_{1}}{2} \sum_{n=0}^{\infty} \sum_{m=-\infty}^{\infty} \frac{g_{n}}{(z-m l)^{n+1}}-\frac{\mathrm{i} \omega_{2}}{2} \sum_{n=0}^{\infty} \sum_{m=-\infty}^{\infty} \frac{h_{n}}{(z-m l+l(d+\mathrm{i} \kappa))^{n+1}}+Q .
$$

Then

$u^{\prime}-\mathrm{i} v^{\prime}=\frac{\mathrm{d}(u-\mathrm{i} v)}{\mathrm{d} z} z^{\prime}-\frac{\mathrm{i} \omega_{1}}{2} \sum_{n=0}^{\infty} \sum_{m=-\infty}^{\infty} \frac{g_{n}^{\prime} \mathrm{e}^{\mathrm{i} m f}}{(z-m l)^{n+1}}-\frac{\mathrm{i} \omega_{2}}{2} \sum_{n=0}^{\infty} \sum_{m=-\infty}^{\infty} \frac{h_{n}^{\prime} \mathrm{e}^{\mathrm{i} m f}}{(z-m l+l(d+\mathrm{i} \kappa))^{n+1}}$,

where

$$
z^{\prime}=R_{1}^{\prime} \zeta\left(1+\frac{a_{1}}{\zeta}+\frac{a_{2}}{\zeta^{2}}+\ldots\right)+R_{1} \zeta\left(\frac{a_{1}^{\prime}}{\zeta}+\frac{a_{2}^{\prime}}{\zeta^{2}}+\ldots\right)
$$


and $g_{n}^{\prime}$, etc., are the perturbations for the vortices (4.1) and (4.2), and are obtained from (4.7), ete. The differentiation with respect to $z$ in (5.7) is done because the velocity has to be evaluated on $|\xi|=1$, and the boundary condition (5.1) will be evaluated in terms of $\zeta$.

The boundary condition is now linear in all the perturbations and solutions are searched for in which all primed quantities are proportional to $\exp (\sigma t)$. If we denote the variables $R_{1}^{\prime}, a_{n}^{\prime}, R_{1}^{\prime *}, a_{n}^{\prime *}, R_{2}^{\prime}, b_{n}^{\prime} R_{2}^{*}, b_{n}^{*}$ by a vector $\phi$, the boundary condition gives an eigenvalue equation of the form

$$
\sigma \boldsymbol{N}(\zeta) \phi=\boldsymbol{M \phi}
$$

where, at least in principle, $\boldsymbol{M}$ and $\boldsymbol{N}$ are known functions of the undisturbed shape and the subharmonic wavenumber $p$. (The infinite sums with respect to $m$ in (5.7) can be done in closed form; see Appendix B.)

Again in principle, the eigenvalue $\sigma$ can be developed as a series in inverse powers of $l$, but the algebraic complexity is fearsome, and numerical methods were employed. Corresponding to a steady solution with some $N$ and $L$, the same truncation was applied to the perturbations, i.e. terms with suffix greater than $N$ were discarded. This gives a column vector with $4 N+4$ rows for $\phi$. The forms like (4.15) were employed for the calculation of the velocity and its perturbation, which leads to an expression of the boundary condition as a series of positive and negative powers of $\zeta .4 N+2$ equations are obtained by equating the coefficients of $\zeta^{n}$ to zero for $n$ in the range $-N \leqslant n \leqslant N$ for each vortex. Two further equations are obtained by fixing the phase by requiring

$$
R_{1}^{\prime}=R_{1}^{* *}, \quad R_{2}^{\prime}=R_{2}^{*} \text {. }
$$

The problem has now become one of calculating the eigenvalues of a generalized complex eigenvalue equation of order $4 N+4$, which can be approached in standard ways. No difficulty was found in accomplishing this.

Some comments are in order. First it will be noted that the boundary conditions for the unsteady calculation are different from those of the steady flow. In the latter case it is necessary to ensure that the equations are independent. This is unnecessary for unsteady calculations, where all that is needed is to ensure that the equations are not inconsistent. Therefore any integral constraint that leads to dependence of steady equations will be automatically satisfied by a consistent evolution equation. We therefore retain the equations that come from the terms independent of $\zeta$ and the coefficients of $\zeta$ and $\zeta^{-1}$ for the second vortex. Satisfaction of the $\zeta$-independent terms is equivalent to conserving area in unsteady flow, and it can be verified from the computer results that all modes with $\sigma \neq 0$ keep the area constant. Indeed this provides a check on the accuracy of the eigenvalues and eigenvectors. As described, the calculation keeps $\omega_{1}$ and $\omega_{2}$ constant. There is a neighbouring equilibrium state in which the velocity of the array is the same $\left(Q\right.$ and $Q^{*}$ are not perturbed) and the strengths and sizes of the vortices are altered. We expect corresponding to this state that there will be two zero eigenvalues in which the eigenfunction does not conserve area. These are indeed found. They could have been eliminated by imposing area conservation to reduce the number of unknowns, but this would have been less convenient. The retention of (5.10) gives two infinite eigenvalues, and it proved easier to make the eigenvector satisfy (5.10) throughout the calculation and actually solve a $(4 N+2)$-order system. This gives $N$ quartets of eigenvalues, one of which is the cooperative mode and the others are the first $N-1$ oscillation modes, and the two neutral modes in which area changes. 
- Secondly, we find perturbations that contain components like $a_{n}^{\prime} \mathrm{e}^{\sigma t+\mathrm{i} m f}$ and $\bar{a}_{n}^{\prime} \mathrm{e}^{\sigma t+\mathrm{i} m f}$ for the $m$ th vortex, and these are not complex conjugates of each other. Toavoid confusion, we denote the perturbation to $a_{n}^{*}$ by $\bar{a}_{n}^{\prime}$. They produce contributions to $z_{m}^{\prime}$ and $z_{m}^{\prime *}$ which are respectively

$$
\begin{gathered}
\frac{a_{n}^{\prime}(f) R_{1} \mathrm{e}^{\sigma(f) t+\mathrm{i} m f}}{\zeta^{n-1}} \\
\widetilde{a}_{n}^{\prime} R_{1}^{*} \zeta^{n-1} \mathrm{e}^{\sigma(f) t+\mathrm{i} m f}
\end{gathered}
$$

Now change $f$ into $-f$ (or $2 \pi-f$ ). We get the contributions to $z_{m}^{\prime}$ and $z_{m}^{\prime *}$ :

$$
\begin{aligned}
& \frac{a_{n}^{\prime}(-f) R_{1} \mathrm{e}^{\sigma(-f) t-\mathrm{i} m f}}{\zeta^{n-1}} \\
& \bar{a}_{n}^{\prime} R_{1}^{*} \zeta^{n-1} \mathrm{e}^{\sigma(-f) t-\mathrm{i} m f}
\end{aligned}
$$

Because of the symmetry expressed by $(2.2), \sigma(-f)=\sigma^{*}(f)$, and $a_{n}^{\prime}(-f)=\left(\bar{a}_{n}^{\prime}(f)\right)^{*}$, where the star here denotes actual complex conjugate. Thus (5.11) is the complex conjugate of (5.14), and (5.12) is the complex conjugate of (5.13). Physically realistic perturbations are therefore obtained by adding (5.11) and (5.13) for the disturbance to $z_{m}$, and (5.12) and (5.14) for the disturbance to $z_{m}^{*}$.

\section{Results}

Steady shapes were calculated for a range of $\alpha$ and $\kappa$ using the method of $\S 4$, and the stability of these shapes to infinitesimal disturbances was calculated using the method of $\S 5$. For the majority of the calculations, the value of $N=8$ and $L=9$ were chosen. Results were checked in two ways. First, calculations were done with $N=16$, $L=17$, and $N=32, L=33$. Secondly, an alternative code which employed the 'interior' mapping of the exterior of the vortex into the interior of the unit circle was employed instead of (4.1). This method was used with $L=N=12$. For $\propto$ less than 0.1 there was no significant difference between any of the results. Calculations using $N=8$ and $L=9$ required about 15 minutes of CPU time for the computation of the steady shapes, and about the same time for the computation of the eigenvalues on a VAX 11/750 computer. Most of the time went for the calculation of the Jacobian and the matrices. A large amount of data was amassed. Here we summarize the salient features.

The behaviour of the shape modes showed no surprises. In fact, the effect of finite area on the oscillation frequencies was generally insignificant. The modes were all stable, and what small change there was due to finite area was consistent with the symmetry requirements.

The interest lay entirely in the behaviour of the cooperative modes, and, in particular, the effect of finite area on the stability boundaries in the $(p, \kappa)$-plane for fixed area. A typical set of results is shown in figure 7. These are for the case $\alpha=0.05$. The ratio of vortex radius to longitudinal separation is $\mathbf{0 . 1 2 6 2}$. The solid lines show the stability boundary in the vicinity of the critical point. The dashed lines are the stability boundary according to equation (3.30) (non-modified) of Kida (1982). It will be seen that the numerical results indicate that the stability boundary is the degenerate case of figures 5 and 6 , in which the hyperbola is a pair of straight lines meeting at the centre $p_{\times}, \kappa_{\times}$. In this case, the dependence of growth rate $\sigma$ on $p$ for 


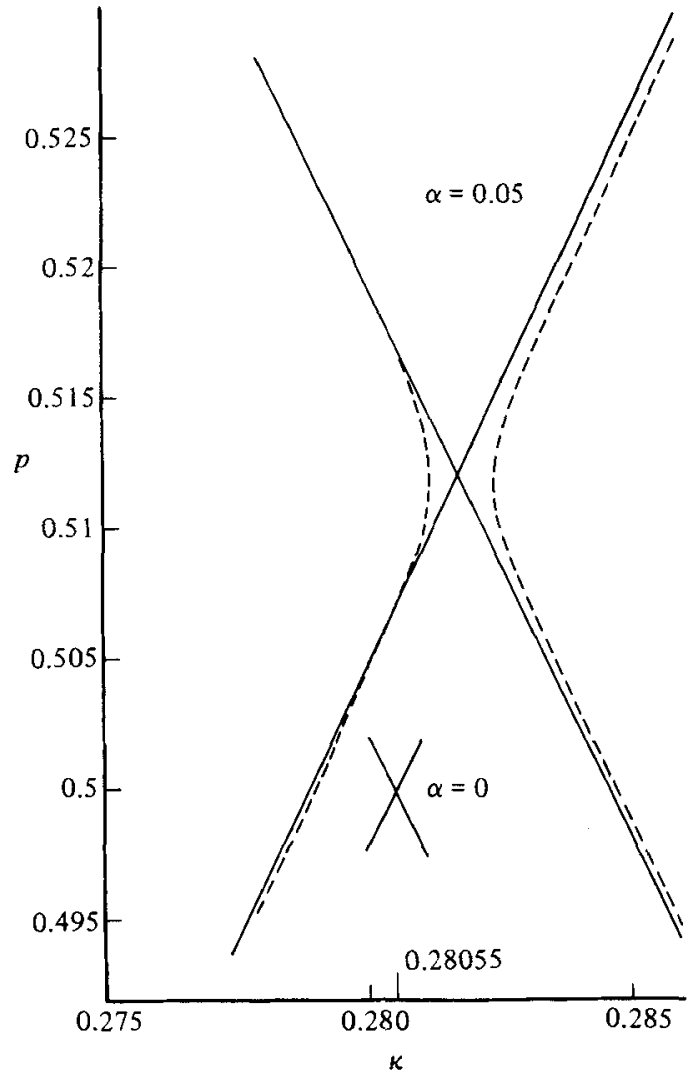

Figure 7. Numerical results for the stability boundary in the $(\kappa, p)$-plane for $\alpha=0.05$. Solid line is result of calculation. Stable regions are above and below the cross. Also shown is the cross for zero $\alpha$. Dashed line is Kida's (1982) result. Kida's modified result agrees with the numerical ones.

$\kappa=\kappa_{\times}$would be the degenerate case of figure 3 in which the ovals shrink to two points at $p_{\times}$and $1-p_{\times}$. The existence of the cross is confirmed by the plot in figure 8 of the difference in the values of $p$ on the stability boundary versus $\kappa$ very close to $p_{\times}$ and $\kappa_{x}$. The numerical points were found to lie on two straight lines which met at a value of the difference indistinguishable from zero. Similar results were found for all other values of $\alpha$ employed. In fact, some runs were done for the relatively large value of $\alpha=0.1854$ (using $N=32$ ), and showed the same behaviour. These results imply that the street is always unstable except for the special value $\kappa=\kappa_{\times}$.

The arguments of $\S 3$ imply that $p-\frac{1}{2}$ and $\kappa_{x}-\kappa_{\mathrm{c}}$ should be proportional to $\alpha^{2}$. Results are given in table 1 and shown in figure 9 . It was found that $p_{\times}^{\prime}$ was closer to quadratic than $\kappa_{\times}^{\prime}$, which deviated considerably for the larger $\alpha$. Assuming to a first approximation that $\kappa_{\times}^{\prime}$ and $p_{\times}^{\prime}$ are quadratic plus cubic in $\alpha$, we have from the results for $\alpha=0.005$ and $\alpha=0.0125$ that $\lambda=0.557$ and $\mu=4.63$. It follows from (3.14) that

$$
c_{3}=-13.6, \quad c_{4}=6.49 .
$$

The existence of the crosses implies that $D=0$, and hence from (3.13)

$$
c_{5}=-11.2 \text {. }
$$

The agreement of these values with those now calculated by Kida (see (3.11)) is very good. 


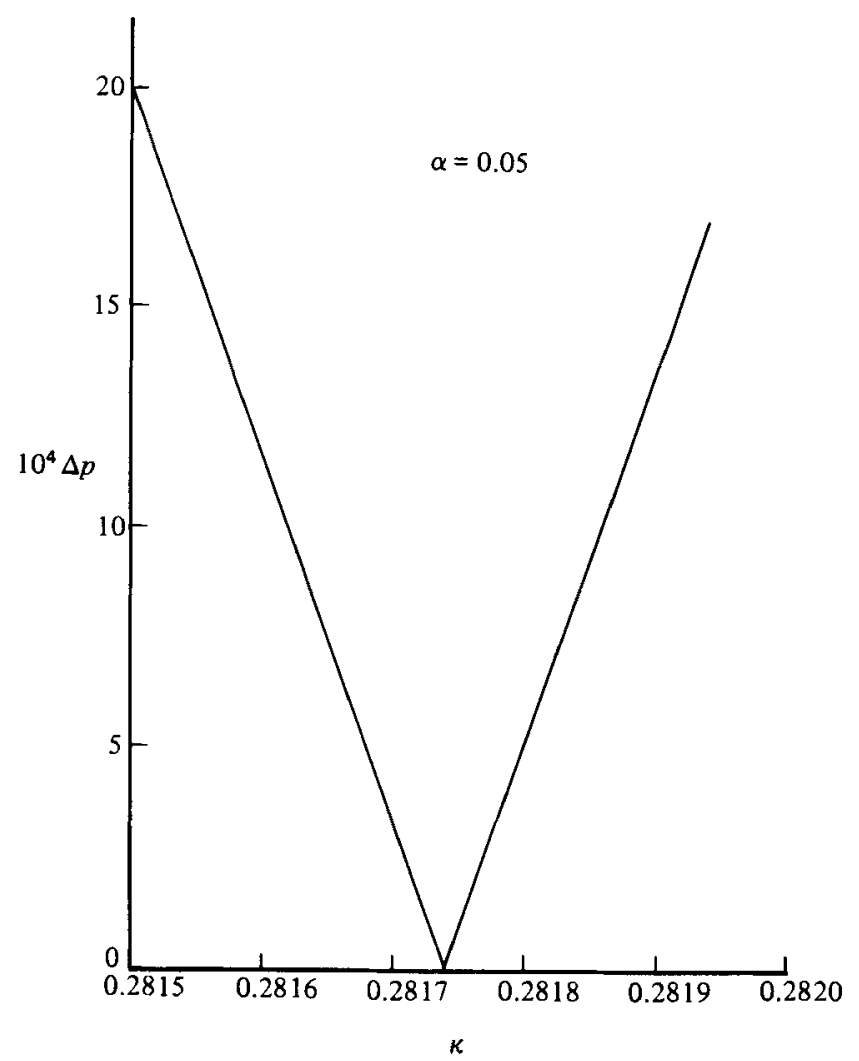

Figure 8. Distance between upper and lower branches of the cross near $\kappa_{\times}$and $p_{\times}$for $\alpha=0.05$. Similar results hold for values of $\alpha$ up to 0.2 .

\begin{tabular}{lllllll}
\hline & 0.005 & 0.0125 & 0.025 & 0.05 & 0.0927 & 0.1854 \\
$\kappa_{\times}^{\prime} 1.38(-5)$ & $8.50(-5)$ & $3.25(-4)$ & $1.19(-3)$ & $3.33(-3)$ & $7.96(-3)$ \\
$p_{\times}^{\prime}$ & $1.16(-4)$ & $7.27(-4)$ & $2.95(-3)$ & $1.20(-2)$ & $4.15(-2)$ & $1.52(-1)$ \\
$\lambda$ & 0.552 & 0.544 & 0.520 & 0.476 & 0.388 & 0.232 \\
$\mu$ & 4.64 & 4.65 & 4.72 & 4.80 & 4.89 & 4.42
\end{tabular}

TABLE 1

A strange property of our results is that there was no sign for any value of $\alpha$ of the crosses breaking up into hyperbolae. This implies that the functions $H_{1}$ and $H_{3}$ are for finite area, and not just to $O\left(\alpha^{4}\right)$, of the form

$$
H(p, \kappa, \alpha)=D_{1}\left(\kappa-\kappa_{\times}\right)^{2}-D_{2}\left(p-p_{\times}\right)^{2},
$$

where $D_{1}$ and $D_{2}$ are functions of $p, \kappa, \alpha$, and $\kappa_{\times}, p_{\times}$are functions of $\alpha$ only. A general result of this kind should be capable of simple proof, and not require extensive computation.

It is known experimentally that the observed values of $\kappa$ in the vortex-street wake of a cylinder increase with downstream distance, as presumably does the area due to viscous diffusion. It would be of interest to determine if the variation of $\kappa$ and $\alpha$ 


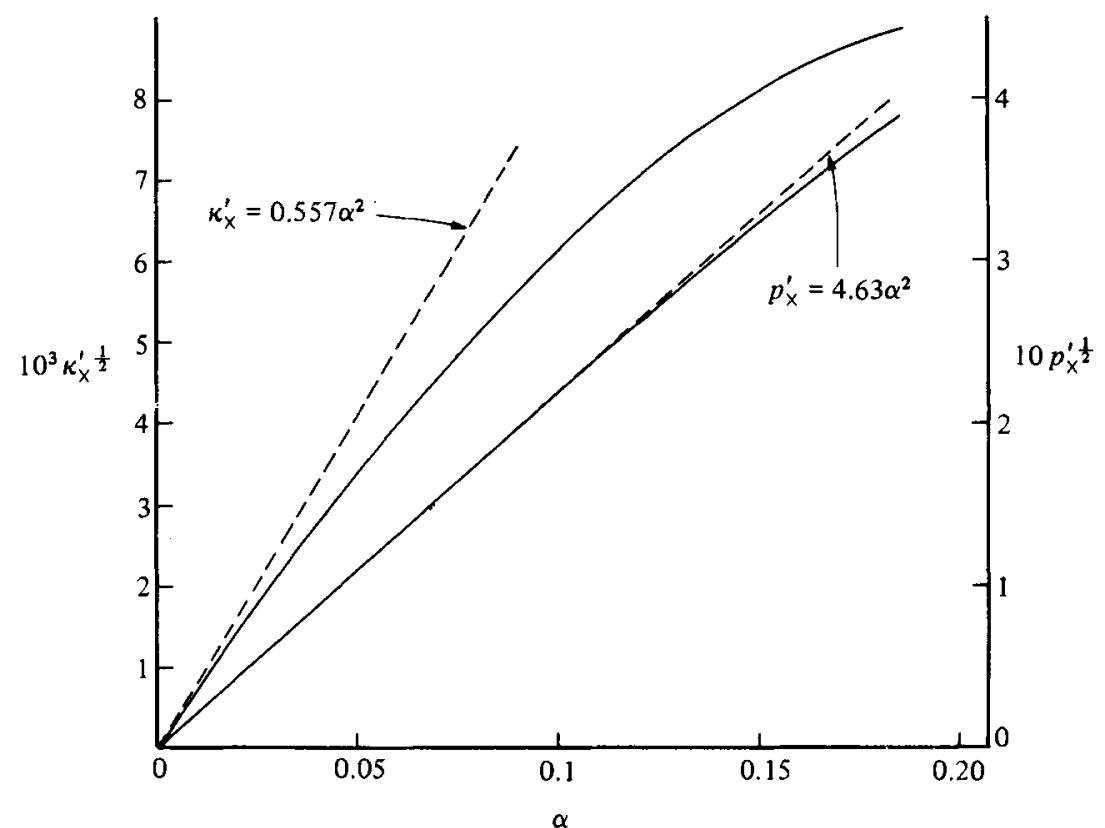

Figure 9. Position of the centre of the cross for various $\alpha$. Solid lines show $p_{\times}^{\prime \frac{1}{2}}$ and $k_{\times}^{\prime \frac{1}{2}}$ versus $\alpha$. Dashed line is tangent at origin.

were such as to keep the street in the state of stability as described by the plot in figure 9 , but this is at present difficult to do as the size of the vortices is not easily estimated from the data.

This work was started while P.G.S. was visiting the Mathematics Research Center, University of Wisconsin, Madison, and continued during a visit to the Mathematics Department, Massachusetts Institute of Technology. He wishes to thank both Institutes for support. The work was also supported by the Office of Naval Research, the Department of Energy (Office of Basic Energy Sciences) and NASA Lewis (NAG 3-179). Ms E. E. Saffman provided extensive help with many of the computations.

\section{Appendix A. Kinematic constraints}

Consider the double row with vortices of strengths $\Gamma_{1}$ and $\Gamma_{2}$ in two parallel horizontal rows. Suppose that the velocity at infinity is $U_{1}, V$ above the top row and $U_{2}, V$ below the bottom row. Conservation of mass requires that $V$ is the same above and below. The equivalence of circulation and vorticity flux gives the relation

$$
U_{2}-U_{1}=\left(\Gamma_{1}+\Gamma_{2}\right) / l
$$

where $l$ is the distance between neighbouring vortices in the same row. We note the vector identity

$$
\boldsymbol{u} \times \omega=\nabla\left(\frac{1}{2} u^{2}\right)-u \cdot \nabla u
$$


Further, for two-dimensional flow, $\omega=\omega k$, where $k$ is normal to the plane. Then, using Green's theorem,

$$
\begin{aligned}
\int \boldsymbol{u} \times \boldsymbol{\omega} \mathrm{d} x \mathrm{~d} y & =-\boldsymbol{k} \times \int \boldsymbol{u} \omega \mathrm{d} x \mathrm{~d} y \\
& =\int \frac{1}{2} \boldsymbol{u}^{2} \boldsymbol{n} \mathrm{d} s-\int \boldsymbol{u}(\boldsymbol{u} \cdot \boldsymbol{n}) \mathrm{d} s \\
& =\boldsymbol{i} V l\left(U_{\mathbf{2}}-U_{1}\right)+\boldsymbol{j} \frac{1}{2} l\left(U_{\mathbf{1}}^{2}-U_{2}^{2}\right),
\end{aligned}
$$

where the integral is over one wavelength of the flow.

Now the left-hand side of (A 3) is proportional in magnitude to the velocity of the vorticity centroid, and this is zero if the flow is steady. Hence in this case either

or

$$
\begin{gathered}
U_{1}=-U_{2}, \quad V=0 \\
U_{1}=U_{2}, \quad \Gamma_{1}=-\Gamma_{2} .
\end{gathered}
$$

The former case is the mixing layer, where the speed of the vortices is the arithmetic mean of the speeds on the two sides. The latter is the wake, where the vortex speed is not the arithmetic mean and the circulations are equal and opposite. These results show that there cannot be a continuous family of steady solutions going from wake to mixing layer with $U_{2}$ changing continuously from $U_{1}$ to $-U_{1}$.

Of importance for our numerical method is the fact that, for the wake where (A 5) holds, the right-hand side of the identity (A 3) vanishes, and hence generally, writing separately the contributions to the left-hand side from vortices in each row, we have

$$
\int_{1} u \omega \mathrm{d} x \mathrm{~d} y+\int_{2} u \omega \mathrm{d} x \mathrm{~d} y=0
$$

which is a consequence of the kinematic relation between velocity and vorticity. Thus, whenever the vorticity field is chosen so that one part of (A 6) vanishes, as is required for the solution to be steady, the other side must also vanish automatically. Hence equating separately to zero the parts of (A 6) in a calculation of steady flow does not give independent equations. This is the justification for dropping the equations for the coefficients of $\zeta$ and $\zeta^{-1}$ on the second-row vortices.

\section{Appendix B. Sum formulae}

The calculation of the steady shapes and stability requires the evaluation in closed forms of the infinite sums

$$
\sum_{m=-\infty}^{\infty} \frac{\mathrm{e}^{\mathrm{i} m f}}{(m+s)^{n}}
$$

where $s$ is an arbitrary complex number, $f$ is an arbitrary real number and $n$ is a positive integer. If $s=0$ the term with $m=0$ in the sum is excluded.

It is convenient to deal separately with $s=0$ and $s \neq 0$. For the first case, we have for $n=1$, by direct summation or the elementary theory of Fourier series,

$$
\sum_{m=-\infty}^{\infty} \frac{\mathrm{e}^{\mathrm{i} m f}}{m}=\left\{\begin{array}{l}
\mathrm{i}(\pi-f) \quad(0<f<2 \pi) \\
0 \quad(f=0 \text { or } f=2 \pi)
\end{array}\right\}
$$

Values outside this range are obtained by applying periodicity in $f$. For $n>1$ we integrate (B 2) $n-1$ times with respect to $f$, choosing the arbitrary constants so that the resulting expression is $2 \pi$-periodic in $f$. 
For $s \neq 0$ we have the formulae

$$
\sum_{m=-\infty}^{\infty} \frac{\mathrm{e}^{\mathrm{i} m f}}{(m+s)}=\left\{\begin{array}{cc}
2 \pi \mathrm{i} \frac{\mathrm{e}^{\mathrm{i} s(2 \pi-f)}}{\mathrm{e}^{2 \pi \mathrm{i} s}-1} & (0<f<2 \pi), \\
\pi \cot \pi s \quad(f=0 & \text { or } \quad f=2 \pi)
\end{array}\right\}
$$

Differentiation $n-1$ times with respect to $s$ gives the sums for arbitrary $n$. Note that it is only for $n=1$ that the sum is not a continuous function of $f$.

The processes of integration and differentiation are easily automated, and can be evaluated by symbolic-manipulation programs.

\section{REFERENCES}

Baker, G. R., Saffman, P. G. \& Sheffield, J. S. 1976 Structure of a linear array of hollow vortices of finite cross section. J. Fluid Mech. 74, 469-476.

Boldman, D. R., Brinich, P. F. \& Goldstein, M. E. 1976 Vortex shedding from a blunt trailing edge with equal and unequal external mean velocities. J. Fluid Mech. 75, 721-735.

DeEM, G. S. \& ZABUSkY, N. J. 1978 Vortex waves; stationary $V$-states, interactions, recurrence and breaking. Phys. Rev. Lett. 40, 859-862.

Doмm, U. 1955 The stability of vortex streets with consideration of the spread of vorticity of the individual vortices. J. Aero. Sci. 22, 750-754.

Havelock, T. H. 1931 The stability of motion of rectilinear vortices in ring formation. Phil. Mag. (7) $11,617-633$.

KIDA, S. 1982 Stabilizing effects of finite core on Kármán vortex street. J. Fluid Mech. 122, 487-504.

Lamb, H. 1932 Hydrodynamics, 6th edn. Cambridge University Press.

Moore, D. W. \& SAFFman, P. G. 1975 The density of organized vortices in a turbulent mixing layer. J. Fluid Mech. 69, 465-473.

Pierrehumbert, R. T. \& Widnald, S. E. 1981 The structure of organized vortices in a free shear layer. J. Fluid Mech. 102, 301-313.

SafFman, P. G. \& Schatzman, J. C. 1982 Stability of a vortex street of finite vortices. J. Fluid Mech. 117, 171-185.

SAFFMAN, P. G. \& Szeto, R. 1981 Structure of a linear array of uniform vortices. Stud. Appl. Maths $65,223-248$. 